\title{
REVERSE OSMOSIS BRINE CONCENTRATION USING FALLING FILM FREEZE CRYSTALLISATION TECHNOLOGY: A PILOT-SCALE STUDY
}

\author{
Mansour Ahmed*, Darren Oatley-Radcliffe ${ }^{\mathrm{b}}$, Paul M. Williams ${ }^{\mathrm{b}}$ \\ ${ }^{a}$ Water Research Center, Kuwait Institute for Scientific Research, P.O. Box 24885, 13109 \\ Safat, Kuwait, mahmed@ kisr.edu.kw \\ ${ }^{\mathrm{b}}$ Centre for Water Advanced Technologies and Environmental Research (CWATER), \\ College of Engineering, Swansea University, Bay Campus, Swansea SA2 8EN, UK, \\ d.l.oatley@swansea.ac.uk, paul.melvyn.williams@swansea.ac.uk
}

*Corresponding author: mahmed@kisr.edu.kw

\begin{abstract}
The reverse osmosis (RO) desalination process has increasingly been utilised with aim of producing drinking water from different marginal sources of water such as seawater, and brackish ground and surface water, due to water scarcity. Unfortunately, desalination applications are limited by the brine disposal challenges including the adverse impact of brine on the surrounding environment. Therefore, this paper is focused on the technical evaluation of falling film freeze crystallisation (FFFC) technology for treating and concentrating RO brine. An industrial pilot plant using the FFFC process was tested and assessed for concentrating RO brine in this study. The experimental results showed that the crystallisation experiments using a feed stage (without the sweating process) and at the operating end-point heat transfer medium (HTM) temperature of $-6^{\circ} \mathrm{C}$, achieved a salt rejection ratio and water recovery ratio of 56.6 and $49.8 \%$ respectively. Whereas at the endpoint HTM temperature of $-24^{\circ} \mathrm{C}$, the salt rejection ratio and water recovery ratio were 24.5 and $84.6 \%$ respectively. The multi-stage process experiments using feed and rectification stages (without the sweating process) achieved a salt rejection and product water recovery ratio of $46.89 \%$ and $64.24 \%$ respectively. By using multi-stage process including feed, rectification and stripping stages (with the sweating process), the salt rejection and product water recovery ratio reached 70.68 and $50.15 \%$ respectively. In general, the results showed that the FFFC technology, using a single freezing stage and without the sweating process, would be an ideal treatment system for concentrating RO brine and to produce saline water to near seawater quality that can be used directly as feed water for a RO plant. The research proved that the investigated FFFC process can be considered as a great solution available for brine concentration and as an alternative for reducing the environmental impact of the large volume of waste streams from coastal and inland desalination plants.
\end{abstract}

Keywords: Freezing desalination technologies, melt crystallisation process, freeze-melting process, reverse osmosis concentrate, reverse osmosis retentate. 


\section{Introduction}

Reverse osmosis (RO) is a pressure driven membrane process and has been widely utilised in seawater desalination applications worldwide. However, RO is not an environmentally friendly process as the RO process discharges high-salinity brine into coastal environments, and the high saline brine from inland desalination plants results in groundwater pollution $[1,2]$. Therefore, RO brine must be disposed in an eco-friendly way to avoid any harm to the surrounding environments. Desalting RO brine is a great challenge worldwide because of its high salinity and high levels of hardness as well as presence of pre-treatment chemicals. In addition, RO brine concentration using conventional desalination technologies may be technically and/or economically non-viable. Therefore, innovative processes and systems for RO brine concentration are substantially needed.

Wang and Chung [3] developed a hybrid desalination process comprising freeze desalination and membrane distillation (FD-MD) processes with aim of using the waste cold energy released from regasification of liquefied natural gas (LNG). The investigated hybrid technologies by the authors was using indirect-contact freeze desalination (ICFD) and directcontact membrane distillation (DCMD) configurations. The DCMD system was utilized for treating the brine discharged from the FD unit. The integrated ICFD and DCMD units was able to produce a drinkable clean water with a total water recovery ratio of $71.5 \%$. In ICFD experiments, the addition of ice nucleate has enhanced the crystallization kinetics in the ICFD experiments. The experimental data showed that the ice salinity tends to be increased with increasing the operation time. This means that the ice salinity is inversely proportional to the cooling rate. In addition to this, the experimentation showed that the investigated FD system is potentially capable of desalting saline water at different salt concentrations. The results obtained from specific energy consumption have verified the advantage of energysaving of the hybrid system. The total energy consumption could be further reduced by using LNG cold energy. The authors recommended in their study to examine a pilot-scale FD-MD combined with a LNG vaporizer, conducting optimization and economic evaluation, and investigating the performance of FD-MD systems for desalinating feed water at different salt concentrations.

Chang et al. [4] investigated and optimized seawater desalination by using indirect contact FD with the aid of cold energy from regasification of liquefied natural gas (LNG). The authors investigated the operating parameters and washing procedure of indirect contact FD using LNG. The investigated operating parameters were coolant temperature, freezing duration, supercooling, seeding, agitation, crystallizer material and subsequent washing procedure on ice production and water quality. Three types of saline waters were investigated as feed in their investigations and these are a $\mathrm{NaCl}$ solution with salt concentration of $3.5 \mathrm{wt} \% \mathrm{NaCl}$, an artificial seawater was prepared based on the formulation of Subow, and a real seawater was obtained from the Public Utilities Board (PUB), Singapore after pre-treatment systems. for the case of $\mathrm{NaCl}$ solution experiments, the experimental results showed that the optimal freezing duration per batch was $1 \mathrm{~h}$ for an iron crystallizer and $1.5 \mathrm{~h}$ for a glass crystallizer. The optimal coolant temperature was found at $8^{\circ} \mathrm{C}$ whereas the optimal amount of washing liquid to purify the ice crystals was around 50 wt $\%$ of the raw ice crystals. Also, the results showed that the investigated system was able to produce a product a drinkable water with water recovery of $25 \mathrm{wt} \%$ and TDS of $300 \mathrm{ppm}$. Both artificial seawater and real seawater were examined under the optimized conditions achieved from $\mathrm{NaCl}$ experiments. The water recovery ratio and salt concentration values were both very close to those obtained from the $\mathrm{NaCl}$ solution experiments. Thus, the process parameters optimized in their study can be applied for the proposed seawater desalination by freezing method. 
Williams et al. [5] introduced and investigated an innovative RO brine concentration system using freeze-melting process based on falling film freeze crystallisation (FFFC) concept. The FFFC system produced a substantial amount of final product water with salt concentration comparable to RO feed and a small amount of highly concentrated RO brine remained. It is reported that the FFFC system is beneficial for concentrating brine from inland RO desalination plants [5].

The freeze-melting process investigated by Williams et al. [5] is classified under melt crystallisation technologies, which are extensively used for the purification of a wide range of chemical substances in chemical processing, pharmaceuticals, and food processing industries [6]. There are two main melt crystallisation processes, namely Suspension freeze crystallisation (SFC) and Solid layer freeze crystallisation (SLFC) [7, 8]. SFC is described in article published by Ahmed et al. [9]. SLFC technologies are classified into two main categories according to operating mode: batch and continuous modes. The SLFC in batch mode uses either a static (e.g. stagnant melt with use of natural convection or forced convection principles) or dynamic process (e.g. flowing melt with use of tube flow or falling film principles) $[7,8]$. The SLFC with a continuous method is either a static (e.g. stagnant melt with use of zone melting or drum crystalliser) or dynamic (e.g. flowing melt with use of belt crystalliser) process [7, 8]. According to Ulrich and Glade [7], the important advantages of SLFC technologies are: (i) incrustation problems are avoided, as these incrustations represent the solid layer, which will eventually be separated, melted, and recovered as final product water; (ii) easily controllable crystal growth rates, due to the driving force being dependant on the temperature difference at the refrigerated surface area of the plate; (iii) a simplified separation process due to absence of an ice slurry. Thus, complicated ice separation and washing equipment, usually used in conventional desalination through freezing processes and melt suspension crystallisation technologies, is avoided. Furthermore, no moving parts are involved in the process equipment (apart from circulation pump); (iv) the operation of the post-crystallisation treatments, such as washing and sweating, are simple; and (v) multistage process design can easily be applied.

The technology used in this article is falling film melt crystallisation and is most commonly used in the separation of organic materials ranging from isomer separation to tar chemical mixtures, and from organics acid to monomers $[10,11]$. Some typical applications are separation of Bisphenol A, acrylic acid, benzoic acid, fatty acids, pharmaceutical intermediate, nitrated aromatics, caprolactam, naphthalene, monochloroacetic acid, palm oil, dichlorobenzene, tetrachlorobenzene, chloro-nitrobenzene, hexachlorobenzene, mono-, di-, and trinitrotoluene, isocyanates, alpha-/beta-naphthol, diphenylphenol, xylenole, p-xylene, aqueous solutions, chlorinated aromatics, p-cresole, p-phenylene-diamine, phenol, and anthracene $[7,10,11]$.

The majority of articles on the FFFC process are focused on process design and operation to improve the purity and yield of crystalline layers [7]. These articles have shed light on a wide range of application areas, including pharmaceuticals, and chemical and food processing [7]. However, there is a marked absence of research in the area of RO brine concentration, which has been overlooked entirely. Accordingly, to investigate the actual performance of the FFFC process for concentrating RO brine, an industrial pilot scale plant was adapted, tested and assessed in this study. The pilot plant used Sulzer FFFC technology, as well as a post-treatment crystallisation system, known as the sweating process. The adopted technology has not previously been tested for saline water applications. As a result, there is no reference data available on the proposed treatment system for saline water applications. Hence, this is the first study to explore and provide quantitative and valuable information concerning the separation efficiency of the adopted process. Indeed, performance cannot be predicted without carrying out experiments, since the actual 
performance of the process depends not only on the physicochemical properties of RO brine (such as equilibrium diagrams), but also on other factors concerning the process operation. Thus, this investigation provides useful and important information on designing a complete commercial plant used as a pre-concentrator system for future application to RO brine concentration. In particular, the tested pilot plant represents a straightforward scale up pilot plant.

The Sulzer FFFC process is characterised as a dynamic process, i.e. flowing melt with use of the falling film principle, in batch operating mode. The Sulzer falling film crystalliser differs from conventional falling film crystallisers and the ice maker machine (including the one that was previously tested and assessed by Williams et al. [5]) in that it distributes the flowing melt and heat transfer medium (HTM) evenly as a falling film (via distribution systems) over the inside and outside surfaces of the tubes, respectively, as illustrated in Figures 1 and 2. This means that neither the shell side nor the inside of the tubes is filled with HTM or melt, but in return, falling film principles are applied instead [7, 8]. Ulrich and Glade [7] mentioned that the refrigerant vapour condenses into a liquid refrigerant, which is distributed on the outside tubes to wet them for refrigerating and warming purposes. This technique enhances the refrigeration method by maintaining a constant temperature distribution along the length of the inside and outside surfaces of crystallizers, leading eventually to obtaining a more homogeneous thickness of ice [7]. Furthermore, this method improves the efficiency of post-purification processes, including the sweating step $[7,8]$.

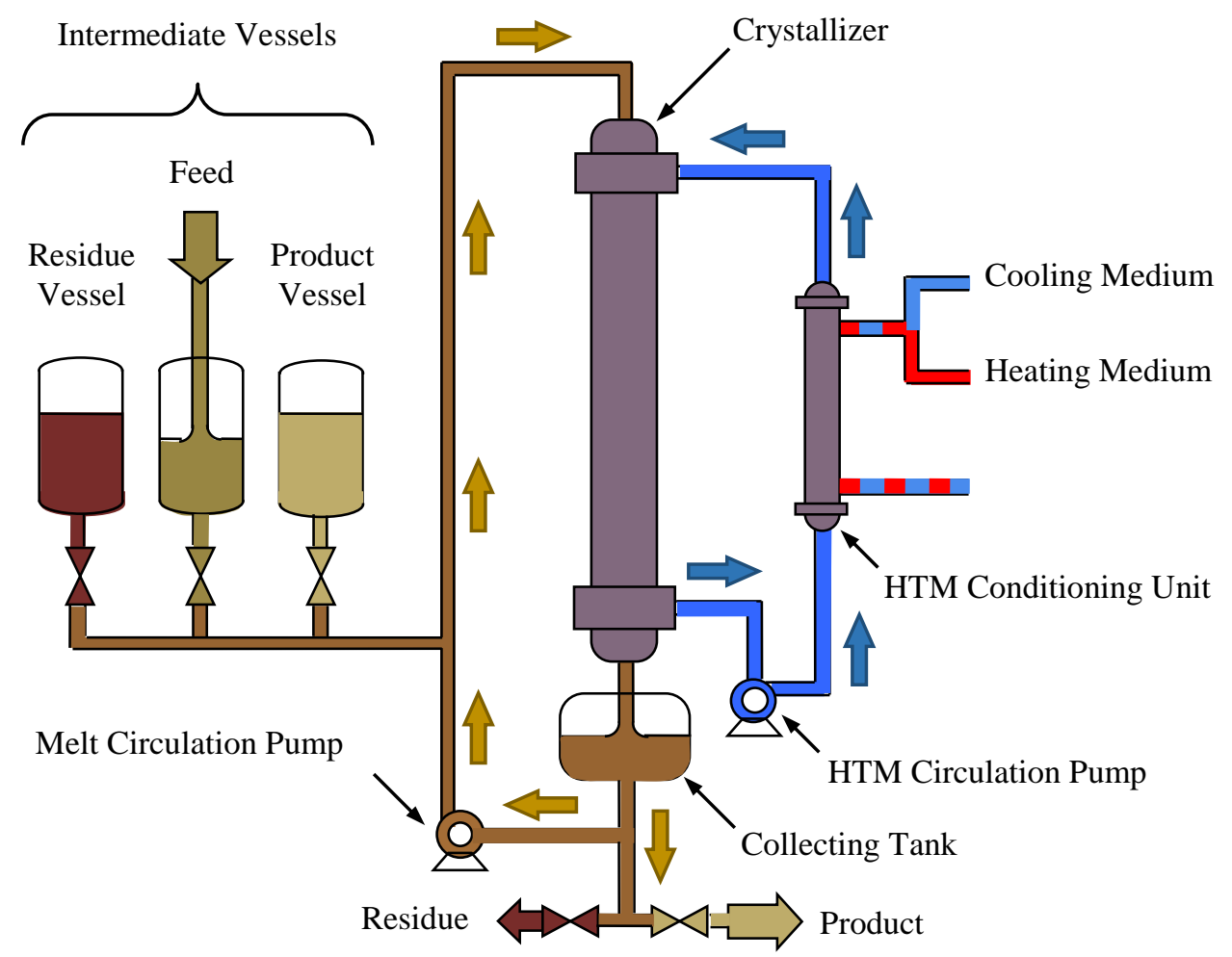

Fig. 1. Typical Sulzer's FFFC plant [8].

A typical example of the Sulzer falling film plant is illustrated in Fig. 1 and consists of a crystalliser, collecting tank, HTM conditioning unit, HTM circulation pump, melt circulation pump, and intermediate storage vessels (which are used for storing feedstock, product, and residue samples). The principles of the Sulzer FFFC process are described in more detail elsewhere [7, 8]. As shown schematically in Fig. 2 the melt and HTM 
distribution systems are installed above the tubes, whereas the tubes are placed above the collecting tank. According to Ulrich and Glade [7], the commercial Sulzer crystalliser consists of several vertical tubes (up to 1,100 tubes) surrounded by a shell. A commercial Sulzer plant containing two crystallisers with 1,100 tubes each means that the diameter of each crystalliser is $4 \mathrm{~m}$, and is capable of producing 100,000 tons of product water annually with purity in the parts per million ranges. Further purification or concentration can be achieved by utilising the intermediate vessel as feed tank and repeating the freezing stage with a single crystalliser. For instance, if the product of the first stage needs to be further purified, then the product vessel of the intermediate vessels will be used as a feed tank to supply the product water of first freezing stage as a feed water in the crystalliser. These steps can be performed in sequence through a single crystalliser using a batch method. These steps will be further described in "2.2 Multi-Stage Design and Basic Operation" sub-section.

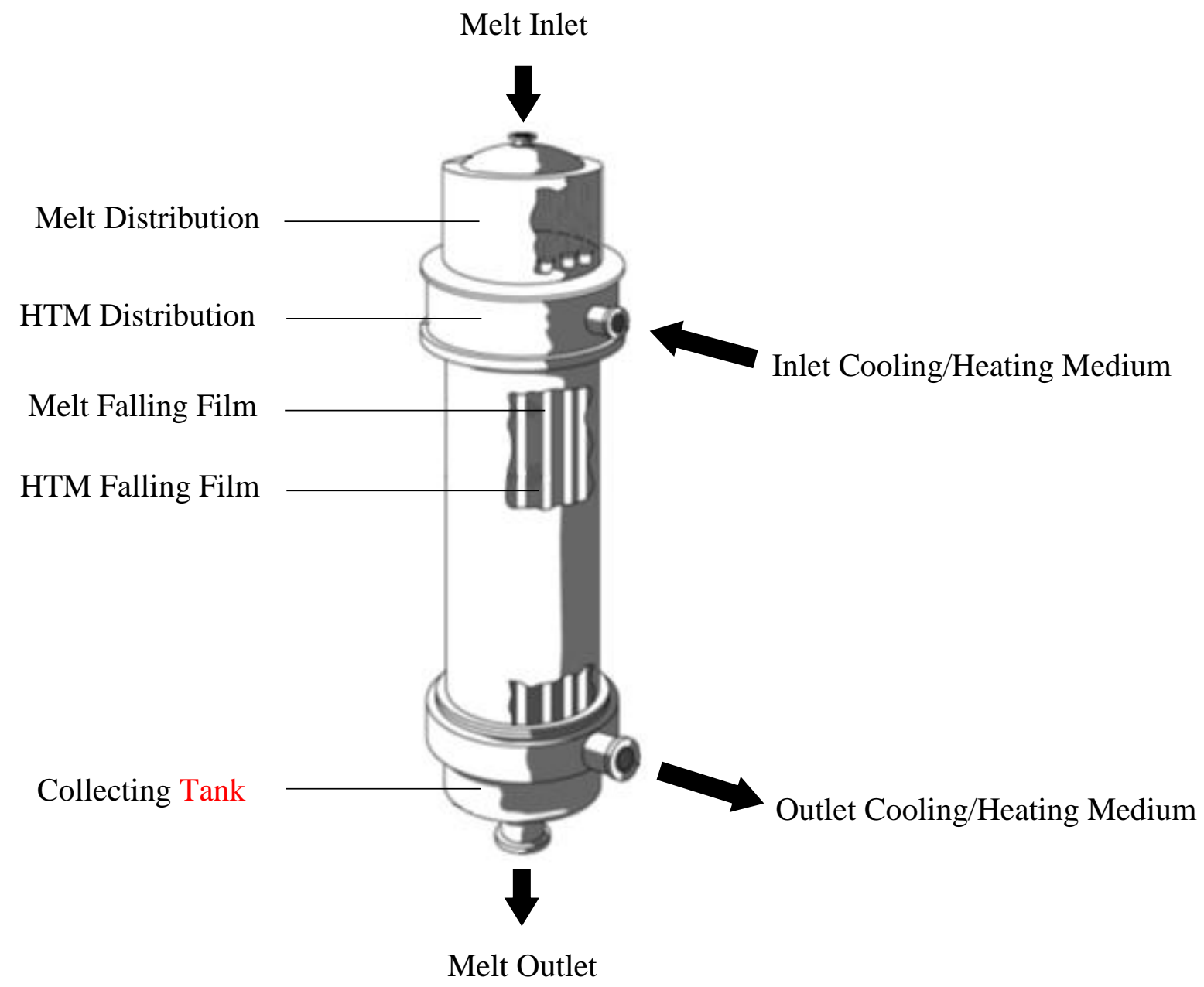

Fig. 2. Typical Sulzer's falling film crystalliser [8].

\subsection{Objectives}

This paper is aimed at evaluating and validating the technical feasibility of FFFC technology developed by Sulzer as a pre-concentration system for concentrating Reverse Osmosis (RO) brine. Several parameters influencing the separation performance of crystallisation and sweating processes, including cooling and heating rates, were investigated in a pilot plant operating in batch mode. Accordingly, the optimal operating limits for key operating parameters in the crystallisation and sweating operations will be presented. Furthermore, the performance of the rectification and stripping stages using the adopted 
technology will be investigated for achieving further purification and concentration of the product and residue, respectively. In addition, the separation effectiveness, under the influence of the FFFC and sweating processes, in the rejection of the major components of ionic concentration (e.g. $\mathrm{Ca}^{2+}, \mathrm{Mg}^{2+},\left(\mathrm{SO}_{4}\right)^{2-},\left(\mathrm{HCO}_{3}\right)^{-}, \mathrm{Cl}^{-}, \mathrm{Na}^{+}$, etc) for the process brine samples will be assessed. Moreover, reference data on the performance of the Sulzer FFFC processes for concentrating RO retentate will be established. This includes a reference physiochemical profile for water samples.

\section{Materials and Methods}

\subsection{Description of the FFFC Process and Basic Operation}

The Sulzer FFFC process is applied under a non-adiabatic environment and operated under atmospheric pressure [12]. The principles of operation of the Sulzer FFFC plant are briefly explained below.

(i) Filling process

Initially, the collecting tank is filled with a predetermined amount of feed by opening the feed valve, whereas the product and residue vales are in the fully closed position as shown in Fig. 1. When the collecting tank is filled with the desired amount of feed, then the feed vessel valve is closed.

(ii) Pre-cooling

Pre-cooling is activated by means of the HTM conditioning unit (using cooling medium) and the melt circulation pump. The melt circulation pump frequently delivers feed from the collecting tank to the melt distribution as shown schematically in Figures 1 and 2. The melt distribution distributes the melt evenly as a falling film over the interior surface of the tube as shown schematically in Fig. 2. When the flowing water passes the interior surface of the tube, the melt is then cooled by means of a concurrent falling film of HTM on the outside surface of a tube [12].

\section{(iii) Nucleation}

In Sulzer system operation, the nucleation process is initiated when the temperature difference between the freezing point of the melt and temperature of the inlet feed reached $10^{\circ} \mathrm{C}$. When the temperature of the inlet feed, which is detected by means of a temperature indicator, had decreased to $7^{\circ} \mathrm{C}$, the nucleation process takes place by deactivating the melt circulation pump, and then rapidly changing the HTM temperature from $0^{\circ} \mathrm{C}$ to the predetermined crystallisation temperature $\left(\right.$ e.g. $\left.-12^{\circ} \mathrm{C}\right)$. Due to the fact that the inside surface of the tube is wet with feed during the pre-cooling process, ice crystals are nucleated and formed from the remaining amounts of the melt adhered in the tube's surface.

(iv) Crystallization

The crystallisation process is achieved by pumping the pre-cooled fluid over the surface of the tube, which includes nucleated ice crystals, to form a thin layer of ice as a cylindrical shell inside the crystalliser. By continuously circulating the cold melt, a thin crystalline layer is allowed to grow inwardly from the refrigerated surface. Consequently, the water level in the collecting tank will decrease as the thickness of the ice layer increases. When the thickness of the ice layer reaches a certain limit (which is dictated by; crystallisation time, crystallisation temperature, salt concentration of the residue, or water level in a collecting tank), then the crystallisation process is deactivated, and the residue is removed from the collecting tank through the residue line. The residue is then either transported to the drain discharge or to an intermediate storage vessel for carrying out multistage operation. This means that the residue can be further treated and concentrated using the intermediate residue vessel as feedstock and repeating the previous steps.

(v) Partial melting (Sweating) 
According to Ulrich and Glade [7], the sweating process is defined as a temperatureinduced purification step based on a partial melting process performed by gradually increasing the temperature of the tube up to a certain level close to the freezing point of the required product. Thus, substantial amounts of impurities adhering to the crystal surface of the crystalline and those brine buckets, contained in pores of the crystalline structure, can be rejected and drained under the influence of gravity [7]. The rejected brines adhering to the crystal surface and those contained within the pores of the crystalline will be partially diluted with pure water melted during the sweating process and then they will be drained under the influence of the gravity. The temperature rise along with the sweating simultaneously reduces the viscosity of the residual liquids and thus further eases the draining process [7]. Therefore, the purity of ice crystals can be significantly improved by the sweating process. On completion of removing the residue from the collecting tank, the partial melting process (i.e. sweating phase) is accomplished by flowing the heating medium into the crystalliser through a HTM conditioning unit. Consequently, the tube wall of the crystalliser is heated gradually to induce partial melting. The melted ice (i.e. sweat fraction) is drained off or transported either to the respective storage vessel or used in multistage operation, for a subsequent purification step. This means that the residue can be further treated by recycling the sweat fraction into the intermediate feed or residue vessel in order to be used as a feedstock and repeating the previous steps.

(vi) Melting

The melting process is operated by increasing the temperature of the heating medium through a HTM conditioning unit. Consequently, the remaining crystal layer is melted and collected as either product or transported into the intermediate storage vessel for a subsequent purification step in multistage operation.

\subsection{Multi-Stage Design and Basic Operation}

Any degree of purity and/or concentration can be obtained by using the intermediate vessel as feedstock and repeating the freezing stage [7, 8]. For Sulzer commercial plants, the number of freezing stages can be varied between one to seven stages [7, 8]. These stages can be performed in sequence through a single crystalliser using a batch method, to reduce the capital and operational costs, however, this option may be limited by several factors, including plant capacity [8].

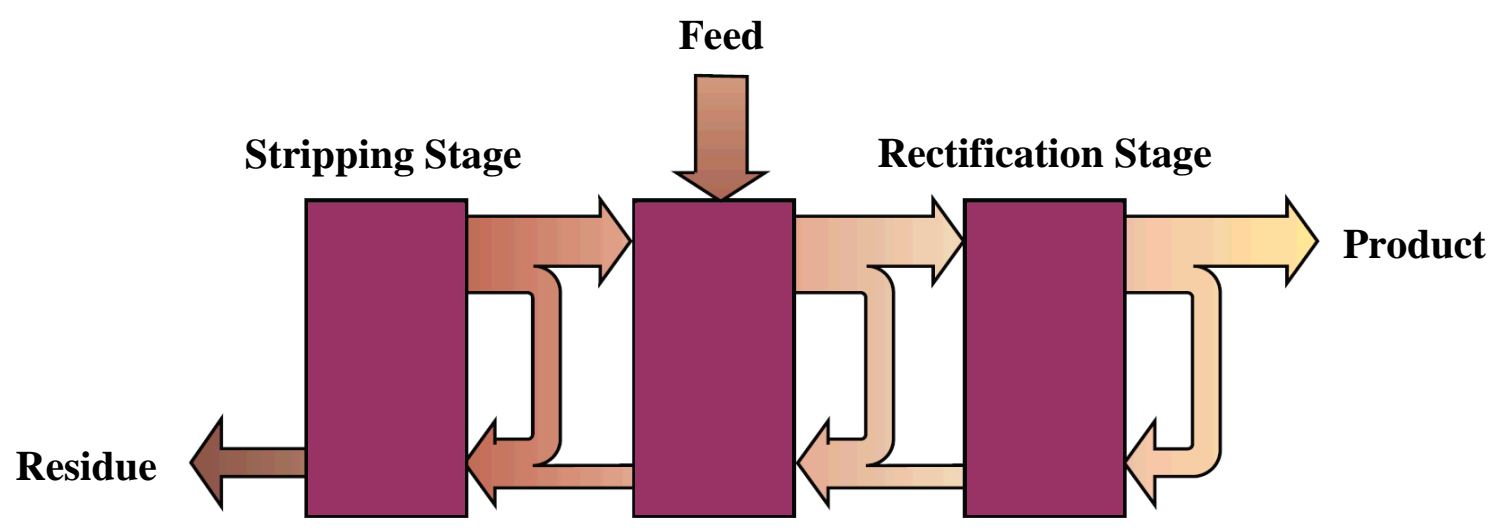

Feed Stage

Fig. 3. Mass flows in a three-stage process [7, 8]. 
Fig. 3 illustrates the mass flow in a three-stage process as an example of multi-stage design. As mentioned previously, the three stages in the process are commonly known as feed, rectification, and stripping stages [7, 8]. The feed stage represents the first freezing stage, which is mainly used for both purifying and concentrating the product and residual liquid, respectively $[7,8]$. The rectification and stripping stages represent the repeated stages for purifying the product and concentrating the residual liquid, respectively $[7,8]$.

\subsection{Preparation of Feed Samples}

One source of actual RO brines was used and tested as a feed-sample in this study. The principles of RO membrane technologies, including the description of reject brines, are described elsewhere $[13,14,15]$. The tested RO brine $(6 \%$ by weight of dissolved salt) was collected from the feed stream and reject brine discharge of the Kadhmah Bottled Water (KBW) plant in Kuwait. Table 1 summarises the results of the major physiochemical characteristics of the tested feed samples i.e. RO brine.

Table 1

Summary of major physiochemical analysis of the tested water samples

\begin{tabular}{llll}
\hline \multirow{2}{*}{ Parameters } & Units & \multicolumn{2}{l}{ Water Samples } \\
\cline { 3 - 4 } & & Arabian Gulf seawater & RO brine \\
\hline Salt Concentration & $\mathrm{wt} \%$ & 4.91 & 6.11 \\
Salt Concentration & $\mathrm{mg} / \mathrm{L}$ & 49,074 & 61,104 \\
Electrical Conductivity & $\mathrm{mS} / \mathrm{cm}$ & 63.6 & 76.6 \\
Freezing Point & ${ }^{\circ} \mathrm{C}$ & -2.52 & -3.1 \\
$\mathrm{Ca}^{2+}$ & $\mathrm{mg} / \mathrm{L}$ & 1,080 & 1,476 \\
$\mathrm{Mg}^{2+}$ & $\mathrm{mg} / \mathrm{L}$ & 1,387 & 1,463 \\
$\mathrm{Na}^{+}$ & $\mathrm{mg} / \mathrm{L}$ & 16,523 & 20,880 \\
$\mathrm{Cl}^{-}$ & $\mathrm{mg} / \mathrm{L}$ & 25,480 & 32,200 \\
$\left(\mathrm{HCO}_{3}\right)^{-}$ & $\mathrm{mg} / \mathrm{L}$ as CaCO & 241.2 \\
$\left(\mathrm{SO}_{4}\right)^{2-}$ & $\mathrm{mg} / \mathrm{L}$ & 3,900 & 4,800 \\
\hline
\end{tabular}

\subsection{Physicochemical Analysis \& Measuring Instruments}

Following collection of feed samples, physicochemical analysis was carried out for the collected sample. After completion of each test, the physiochemical analysis was then carried out for the product (i.e. melted ice crystals layer) and residue samples. The physiochemical analysis of water samples included measurements of the temperature, Total Dissolved Solids (TDS), electrical conductivity, $\mathrm{pH}$, volume, and weight. Two different types of salinity measurements are considered in the physiochemical analysis, in order to ensure and check the results of the salinity measurements; (i) electrical conductivity and (ii) gravimetric method. The accuracy of salinity measurements (which are obtained by a gravimetric method) was also ensured by using a simple mass balance equation. In addition, full water chemistry analysis was conducted for all samples of each experiment. The purpose of conducting full water chemistry analysis is to detect the major components of ionic 
composition found in the feed, product, and residue. The reliability of full chemical analysis of the water samples was ensured by using a charge balance error. Furthermore, the salinity, in term of TDS, was also ensured by comparing the results of TDS obtained through physicochemical analysis and gravimetric method. In addition, the measurements of freezing point were covered in this investigation. Furthermore, the temperature profiles and running times of the pre-cooling, crystallisation, partial melting, total melting, and freezing stage of each test were monitored and recorded. However, the $\mathrm{pH}$ measurements were not considered in this study. The physicochemical analysis of water samples was performed experimentally using the measuring instruments, auxiliary equipment, tools, and laboratory facilities of Sulzer Chemtech Ltd (Buchs SG, Switzerland). For further investigation and cross checking, several sets of water samples were sent to two different laboratories at KISR (Kuwait) namely Desalination Research Plant (DRP) laboratory and the Central Analytical Laboratories (CAL) for performing similar chemical analysis and also carrying out full chemical analyses on water samples to detect the major ionic composition. Results of these measurements are tabulated in the results and discussion section.

The salt concentration of the feed sample was measured via a conductivity meter (Cond 3110, Tetra Cond 325) and a conductivity probe (Conductivity probe model: TetraCon 325, Wissenschaftlich-Technische Werkstätten $\mathrm{GmbH}$ ) to ensure that the correct concentration was achieved. The measurements of TDS were experimentally determined, using the gravimetric method, by evaporating a known weight of the water sample to dryness, and weighing the solid residue. The equipment involved in obtaining gravimetric measurements are an oven (Heraeus instruments, Type: UT 12P, D-63450 Hanau, Kendro Laboratory Products), Petri dish, and laboratory balance (Mettler PM 460, Delta Range, CH8606). The accuracy of salt concentration results was assured through a simple mass balance equation. The volume and weight measurements, on the other hand, were determined by a laboratory beaker, scaled borosilicate glass cylinder (Barcode: CYL-350-020J, Fisher Scientific) and a laboratory balance (Mettler Toledo, Model: PM30-K). The running time for the crystallization process was measured by a stopwatch timer (HS-10W Stopwatch, Casio).

The freezing points were experimentally measured with an instant digital thermometer (P650 series, Serial No.: 65006040472) and a temperature sensor (Pt100, IEC A 240119-1), while the refrigeration system consisted of the following equipment; (a) thermostatic bath (Haake C, Type: 001-0505, Nr: 840096), (b) PID temperature controller (Haake PG 40, Type: 000-9030, Nr: 830264), (c) bath circulator (Haake F3, Type: 000-9601, Nr: 840117), and HTM, which is a mixture of ethylene glycol and deionised water. On the other hand, a DR 5000 Spectrophotometer (Hach, DR 5000) was used to detect the major components (ionic composition) of the feed sample.

\subsection{Pilot Scale Experimental Setup}

Figures 4 and 5 show the investigated Sulzer FFFC pilot test unit. This pilot plant is used by Sulzer for investigating new fields of application. The pilot plant is completely equipped with all necessary measuring instruments and control devices. All equipment of the pilot plant in contact with cooling/heating medium and investigated feed are thermodynamically insulated from the surrounding environment. The tested pilot plant is a straightforward scale up pilot plant where a commercial scale crystallizer may be built by adding a number of tubes of the same size of the length and inner diameter of the tested crystallizer's tube. The tested pilot plant provides a higher specific production rate, in terms of water recovery ratio, within a single-stage process. In addition, the examined pilot plant provides a greater operating depth in different key operating parameters for both crystallisation and sweating operations. The pilot plant is provided with a desktop computer 
and Strip Chart Recorder (SCR) (Eurotherm Chessell, Model No.: 4180M) as illustrated in Figures $6 \mathrm{a}$ and $6 \mathrm{~b}$, respectively. The desktop computer is provided with a software package for leading and controlling the operation of the pilot plant. The package monitors the operational status and records the experimental data, such as the measurements of running time, melt temperature, HTM operating temperature, and flow rate of melt circulation pump throughout the freezing stage, which includes pre-cooling, crystallisation, partial melting, and total melting processes. At the same time, some of the majority of the key operating parameters were controlled through a software package installed in a computer-controlled system connected to the pilot plant. The strip chart recorder data acquisition system, on the other hand, is used for continuous tracking of the process, printing the trends of temperature profiles of melt and HTM throughout the freezing stage.

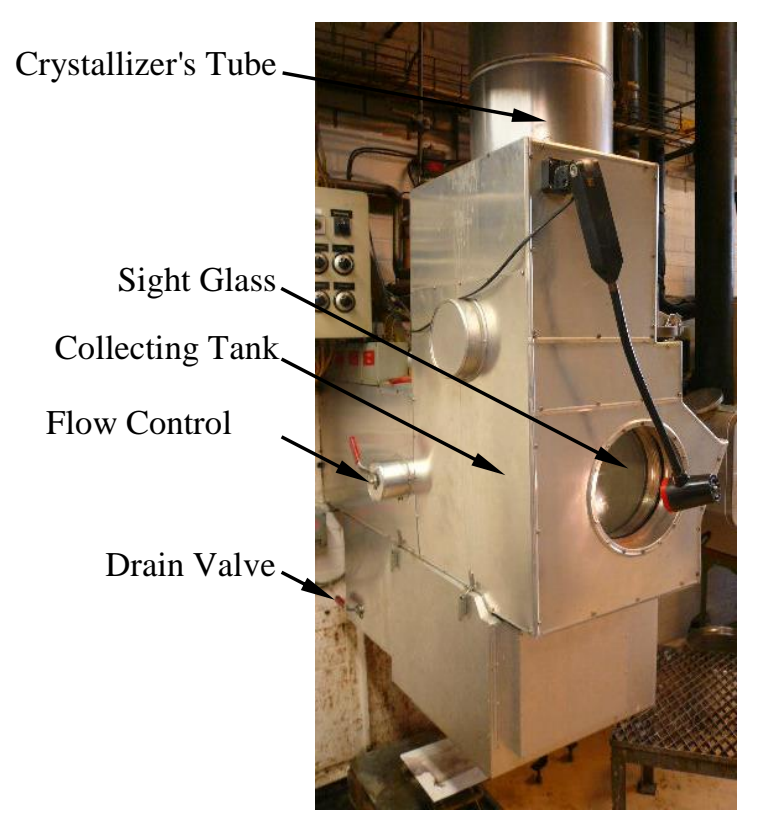

(a) Left-hand side view of pilot plant

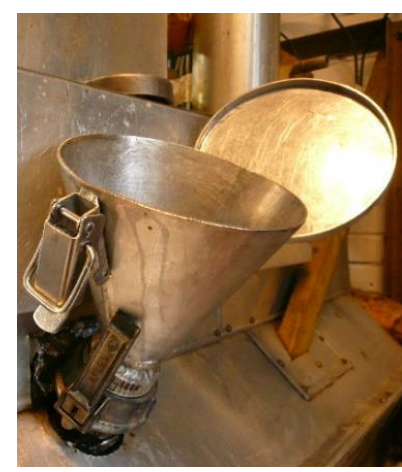

(c) Funnel

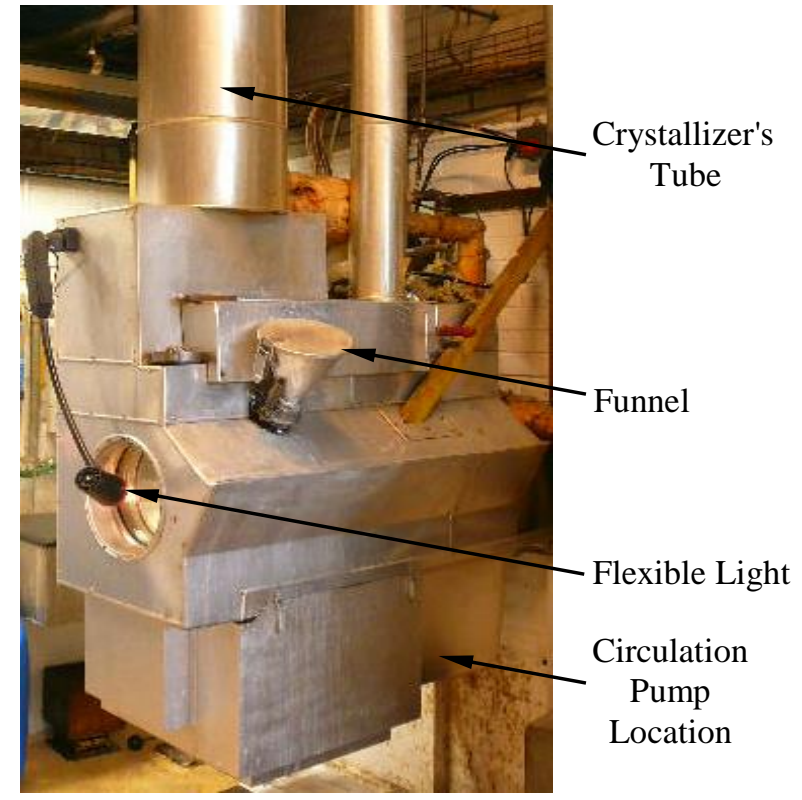

(b) Right-hand side view of pilot plant

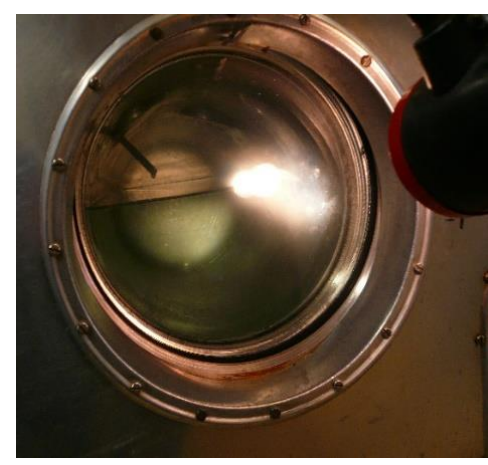

(d) Sight glass \& water level Indicator 


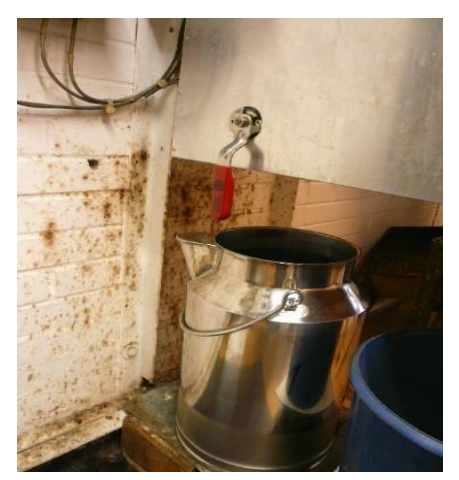

(e) Drain valve (i.e. sampling point)

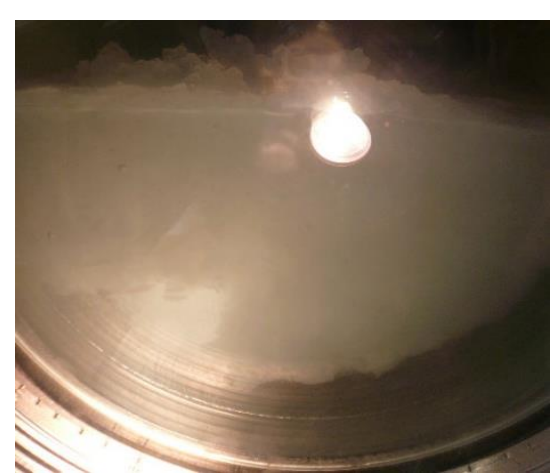

(f) Monitoring ice suspension

Fig. 4. Tested Sulzer FFFC pilot plant.

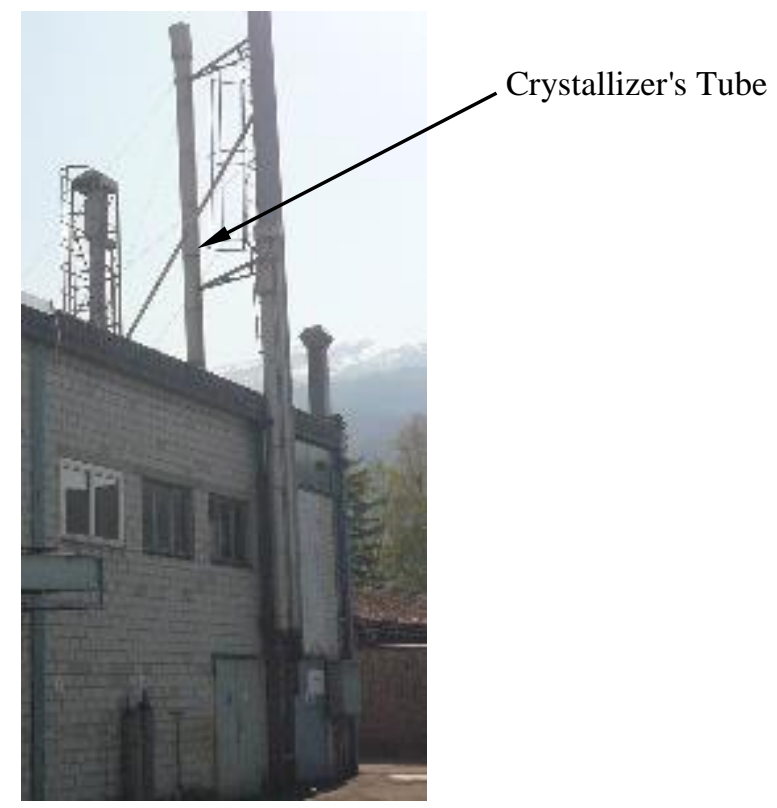

Fig. 5. Outside-view of the tested pilot plant.

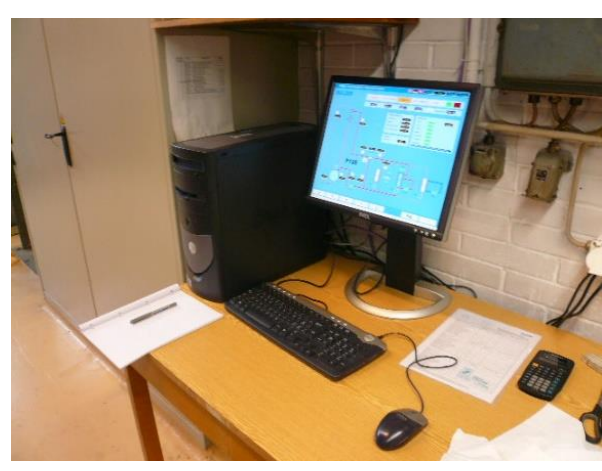

(a) Desktop computer

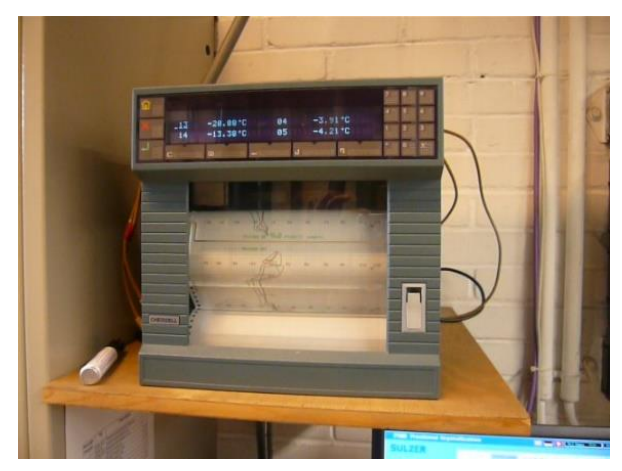

(b) Strip chart recorder (SCR)

Fig. 6. Apparatus for controlling and maintaining the pilot plant.

Fig. 7 shows that the tested pilot plant consists of the following equipment; collecting tank (T-1), crystalliser's tube (S-1), melt circulation pump (P-1), flow control valve (FCV), drain valve (DV), funnel (F), temperature indicators (i.e. (TI-101), (TI-102), (TI-204), and (TI-205)), HTM circulation pump (P-3), and HTM conditioning units (which are used for supplying cooling medium (C-1) and heating medium (H-1)). The pilot unit is provided with 
a single-tube falling film crystalliser as shown in Figures 4a, 5, and 7. This crystalliser is installed above the collecting tank, and consists of a single vertical tube, and two distribution systems for the melt and HTM. The length and inner diameter of the tube are $12 \mathrm{~m}$ and 70 $\mathrm{mm}$, respectively.

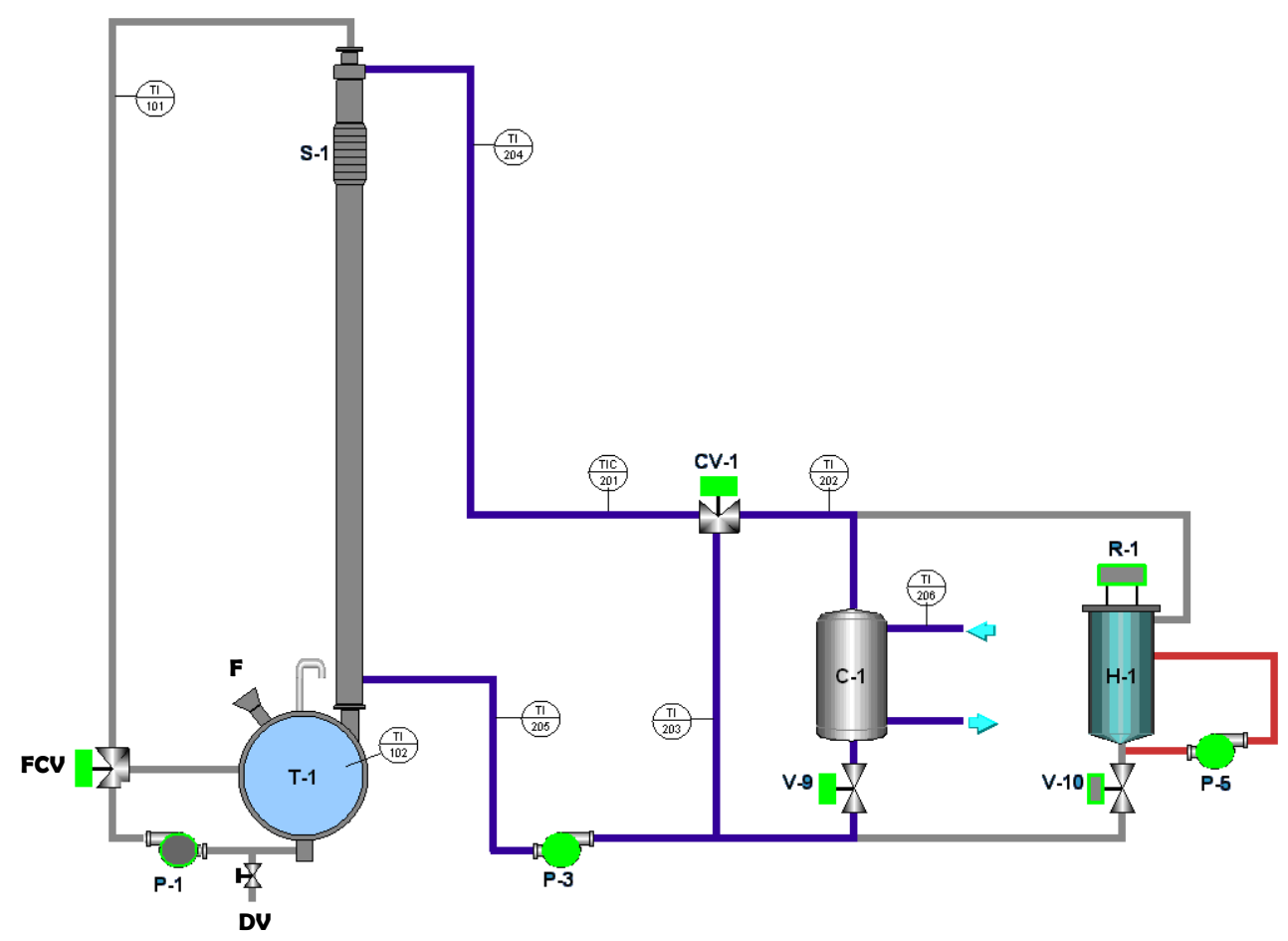

Fig. 7. Process flow diagram of the pilot-plant, adapted from screenshot of the pilot plant software package.

As illustrated in Figures $4 \mathrm{a}$ and $4 \mathrm{~b}$, the collecting tank is provided with the following: (i) A reservoir with a capacity of $35 \mathrm{~L}$ (see Fig. 4a), which is joined with a crystalliser tube, where the reservoir is positioned below the tube; (ii) A funnel is attached with a lid (see Fig. $4 \mathrm{c}$ ), which is used for facilitating the filling process. (iii) A sight glass with a water level scale which enables the user to visually monitor the return flow behaviour inside the collecting tank, since the pilot plant is considered a closed system. Throughout the freezing stage, the sight glass helps detect the water level (see Fig. 4d), and visually observe the appearance of the undesired ice suspension inside the collecting tank (see Fig. 4f). The sight glass is installed in front of a collecting tank as shown in Figure 4a. (iv) A flexible light (see Fig. 4b) is installed on the front side of collecting tank (above the sight glass) to illuminate the inside of the collecting tank to visually monitor the water level, and the appearance of the ice suspension as stated previously. (v) A resistance wire (i.e. heat source element), which is wrapped around the collecting tank in order to control the feed temperature inside the tank throughout the operation of the freezing stage. The thermal energy provided by the resistance wire is controlled by a computer. The flow control valve (see Fig. 4a) is a hand-operated three-way ball valve and is used for controlling; (i) the flow rate of the falling film of the melt inside the tube, and (ii) the flow rate of the circulated feed into the collecting tank through a bypass pipeline. The pilot plant also has a drain valve, which is a hand-operated ball valve installed beneath the collecting tank, as illustrated in Figures $4 \mathrm{a}$ and $4 \mathrm{e}$. The purpose of this valve is to remove (or collect) the residual feed, sweat fractions, and product sample from the collecting tank under the influence of the gravity. This valve is used as the sampling point. 


\subsection{Experimental Procedure}

The operating procedures for crystallisation and sweating experiments using the mentioned pilot plant are shown schematically in Fig. 8. These experiments were carried out in batch mode. Before performing the first experiment of each day, the main refrigeration system was initially operated to cool down the refrigeration unit. The physiochemical analyses were initially performed on the feed sample. Before starting the experiment, a predetermined weight of feedstock was experimentally measured by means of a laboratory balance. The collecting tank was manually filled with the predetermined amount of feed. Following this step, the refrigeration system was activated. The pre-cooling, crystallisation, partial melting, and total melting processes were operated in sequence order for all tests.

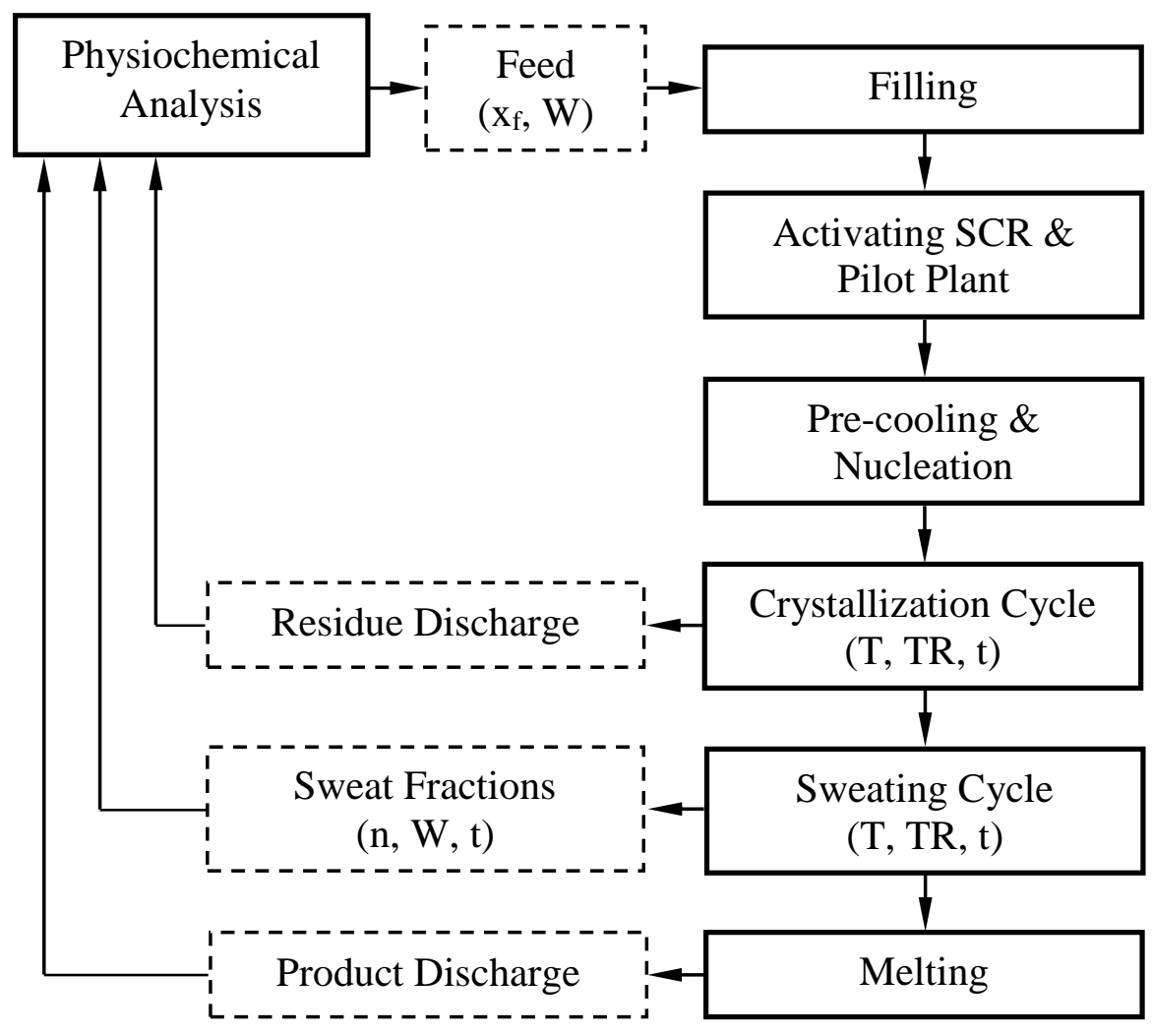

Fig. 8. Simplified block diagram of the operational process for the experimental set-up, where $\left(\mathrm{x}_{\mathrm{f}}\right)$ is the feed concentration $(\mathrm{ppm}),(\mathrm{W})$ is the sample weight $(\mathrm{kg}),(\mathrm{t})$ is the running time (minute), $(\mathrm{T})$ is the temperature $\left({ }^{\circ} \mathrm{C}\right),(\mathrm{n})$ is the number of sweat fractions, and (TR) is the temperature ramp.

The actual operating period of the crystallisation process in the experiments was varied from 23.3 to 120 minutes. The crystallisation experiments were carried out at different operating HTM temperatures, ranging from $-6^{\circ} \mathrm{C}$ down to $-28.4^{\circ} \mathrm{C}$, at various HTM cooling rates varied from 0 to $-0.75^{\circ} \mathrm{C} / \mathrm{min}$. These experiments were carried out at maximum water recovery ratio, which was dictated when the water level in the collecting tank reached the minimum allowed limit, i.e. $5 \mathrm{~L}$. One point to note here is the use of a cooling rate of $0{ }^{\circ} \mathrm{C} / \mathrm{min} .0{ }^{\circ} \mathrm{C} / \mathrm{min}$ is just a value that can be inserted in the computer. In practice, the cooling rate will not be exactly $0{ }^{\circ} \mathrm{C} / \mathrm{min}$, but it will be below this value and it can be theoretically calculated by the following term:

$$
\text { Cooling Rate }=(\text { End-point temperature }- \text { Start-point temp }) / \text { running time }
$$


Experimental data were gathered and analysed to provide quantitative information on the separation performance of this technology for concentrating RO brines. Throughout the experimental investigations, no pre-treatment system or chemical additives were considered for use, either before or during the tests. For clarity, the HTM temperature of the crystallisation process is identified, and referred to, in the course of this paper, as the crystallisation temperature.

Throughout the experiments, the formation of an ice suspension inside the collecting tank was eliminated by applying one of the following methods; (i) reducing the flow rate of the flowing film inside the crystalliser tube via the pumping power ratio setting. (ii) Reducing the flow rate of the flowing film inside the crystalliser tube by adjusting the flow control valve. (iii) Increasing the setting of the tank heating power. This action helps warm the collecting tank in order to eliminate the formation of an ice suspension by melting. (iv) Increasing turbulence inside the collecting tank through use of a bypass valve (i.e. flow control valve). This can be accomplished by increasing the flow rate through the pumping power ratio setting, and simultaneously adjusting the bypass valve.

For the purpose of establishing a phase diagram for the tested feed materials, and instantly estimating the results of TDS and freezing point by means of electrical conductivity, the parameters of salt concentration (measured in wt $\%$ and TDS), and freezing point, were measured experimentally over a wide range of electrical conductivity, ranging from $424 \mu \mathrm{S} / \mathrm{cm}$ up to $220 \mathrm{mS} / \mathrm{cm}$.

\subsection{Results and Discussion}

The variations of the experimental results of key parameters were plotted on graphs as shown in Figure 9. Based on the experimental results, the empirical polynomial correlations were derived and fitted for the freezing point as a function of TDS value (ppm), salt concentration (wt\%), and electrical conductivity $(\mathrm{mS} / \mathrm{cm})$. Furthermore, the empirical polynomial correlations were also derived and fitted for the electrical conductivity $(\mathrm{mS} / \mathrm{cm})$ as a function of salt concentration (wt \%). These equations were also used to instantly determine the results of the main key parameters through the conductivity measurement.

Fig. 9a show the experimental phase diagram, and empirical graph and equation (including $\mathrm{R}$ value) for the AG seawater and RO brine. The important observations that can be revealed from the phase diagram are as follows: (i) the freezing points of AG seawater and $\mathrm{RO}$ brine were $-2.4^{\circ} \mathrm{C}$ and $-3.1^{\circ} \mathrm{C}$ respectively. (ii) the eutectic temperature for the $\mathrm{AG}$ seawater and RO brine was $-20.45^{\circ} \mathrm{C}$, which was obtained at TDS value, electrical conductivity, and weight ratio of $238,560 \mathrm{ppm}, 220 \mathrm{mS} / \mathrm{cm}$, and $23.85 \mathrm{wt} \%$, respectively.

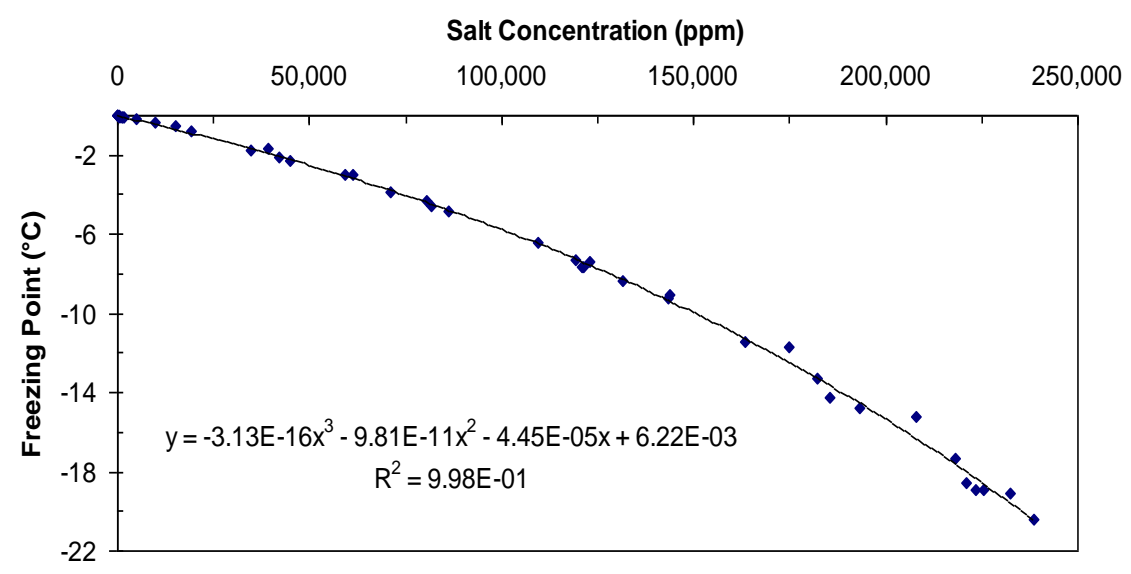

(a) Phase diagram 


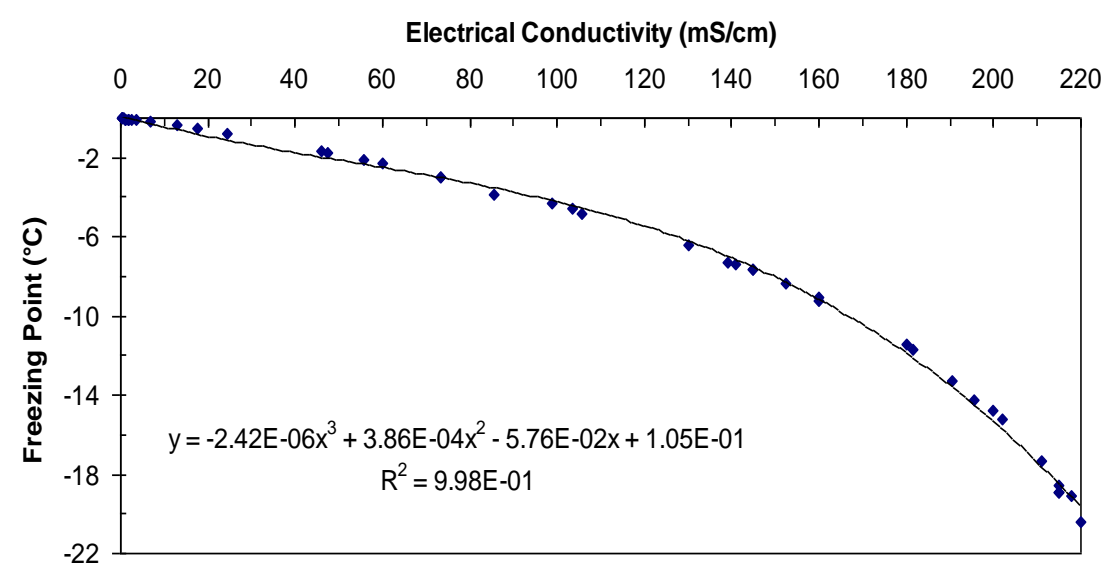

(b) Conductivity vs. freezing point

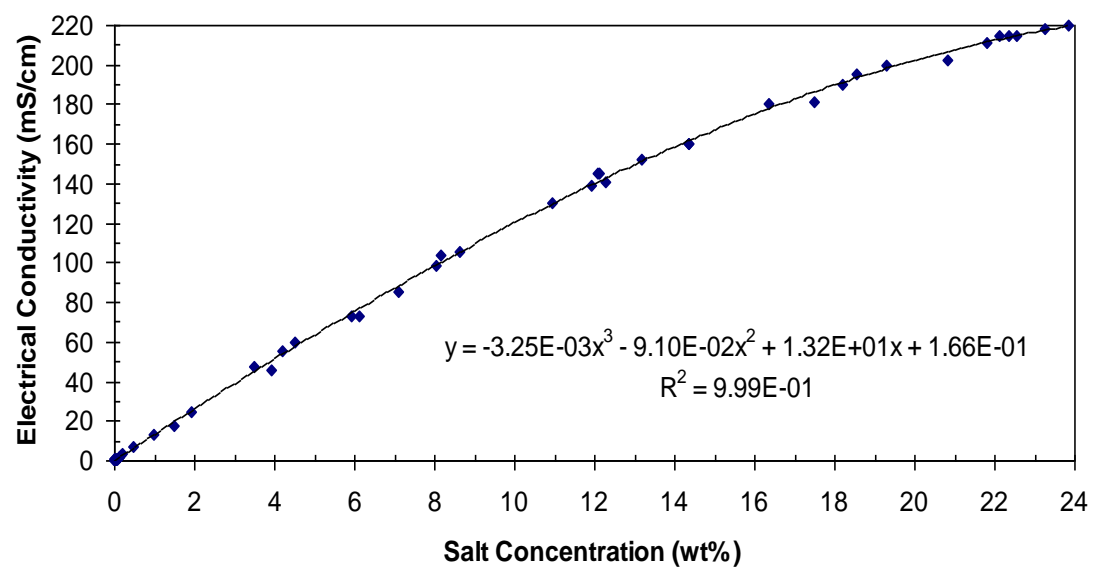

(c) Salt concentration vs. conductivity

Fig. 9. Summary of the phase diagram and relationships between the main key parameters for the AG seawater and RO brine, where; $\mathrm{y}$ and $\mathrm{x}$ are the dependant and the independent variables of the empirical equation respectively, and $\mathrm{R}^{2}$ is the polynomial regression correlation coefficient.

\subsection{Crystallisation experiments using feed stage (without the sweating process)}

In the first series of experiments, the potential of the Sulzer FFFC process (without use of the sweating process) was investigated for concentrating and treating RO brine. These experiments were carried out in a feed stage process, i.e. single freezing stage. The influence of several parameters, namely crystallisation temperature and time, cooling rate, feed concentration, and average growth rate on the salt rejection and water recovery ratio were examined. Results of salt concentration of product, salt rejection, water recovery, and crystallisation time as a function of the end-point cooling HTM temperature and rate are shown in Figures 10a-10e.

Fig. 10a shows the variation of the salt concentration of product water, as a function of the end-point cooling HTM temperature at different cooling rates. A clear tendency of decreasing salt concentration in the product water with rising end-point crystallisation temperature and cooling rate can be observed. The results indicate that the adopted process is sensitive to the variations of end-point cooling HTM temperature from -6 to $-18^{\circ} \mathrm{C}$ as shown in Fig. 10a. However, no significant change in the salt concentration of product water was 
observed when the end-point cooling HTM temperature was reduced from $-18^{\circ} \mathrm{C}$ down to $28.4^{\circ} \mathrm{C}$, where the product concentration stabilised at an average value of $4.5 \mathrm{wt} \%$ as shown in Fig. 10a. This indicates that the tested pilot plant, at lower operating temperature and cooling rate limits, was capable of providing product water at lower salt concentration than that of seawater, as shown in Fig. 10a. This means that the tested process becomes insensitive to the end-point cooling HTM temperature and cooling rate as long as the desired product concentration is closer to the RO feed. Also, Fig. 10a shows that the maximum and minimum salt concentration of product water sample was 4.61 and $2.65 \mathrm{wt} \%$ respectively. This means that the salt concentration of product water measured is lower than the salt concentration of RO feed at all times, as indicated in Fig. 10a. The behaviour of the treatment system for the given conditions was insensitive to a wider range of crystallisation temperatures when the desired quality of product water is equal to that of RO feed. The results suggested that the commercial plant using the adopted crystalliser can be technically designed and built with a wider range of crystallisation temperatures and cooling rates for RO brine concentrations. This includes lower operating limits to produce a substantial amount of the desired quality of product water (i.e. RO feed quality), leading eventually to a significant decrease in the capital costs. However, designing commercial plants with lower crystallisation temperatures might be limited by a significant increase in the power consumption. Thus, the capital and operational costs must be optimised prior to that, in terms of the final decision on plant capacities and conditions, including crystallisation temperature and cooling rate. In general, the crystallisation experiments, which were carried out under different ranges of crystallisation temperature and cooling rate, suggested that the adopted technology is feasible for concentrating RO brines and simultaneously producing saline water of near seawater salt levels that can be recycled to a conventional desalination plant.

Fig. 10b shows the effect of the end-point cooling HTM temperature with different cooling rates on salt rejection ratio. The influence of end-point cooling HTM temperature on the salt rejection is similar to that observed for product concentration, since the results depend mainly on the feed and product concentrations. The salt rejection ratio is proportional to the end-point cooling HTM temperature and cooling rate of HTM, as shown in Fig. 10b. The maximum and minimum salt rejection ratios were $56.6 \%$ and $24.5 \%$ respectively, achieved at operating end-point HTM temperatures of -6 and $-24^{\circ} \mathrm{C}$ respectively. These results were obtained at a cooling rate of $0^{\circ} \mathrm{C} / \mathrm{min}$. For experiments with lower cooling rates, there was no significant change in the salt concentration of product water observed, and the averaged value of the salt rejection was $25 \%$, as shown in Fig. $10 \mathrm{~b}$.

Fig. 10c shows the influence of end-point cooling HTM temperature with different cooling rates on the water recovery ratio. The water recovery ratios were found to be inversely proportional to the end-point cooling HTM temperature and cooling rate. The minimum and maximum water recovery ratios were $49.8 \%$ and $84.6 \%$ respectively, achieved at operating end-point HTM temperatures of -6 and $-24^{\circ} \mathrm{C}$ respectively. The calculations of the maximum water recovery ratio for RO feed quality product water gave about $92 \%$, which, in practice, can be obtained easily through the tested pilot plant. This is because the tested crystalliser is capable of producing a crystal layer with a maximum volume of $46 \mathrm{~L}$ (exceeding the total volume of collecting tank), when the crystalliser is completely filled with cylindrical ice. Nevertheless, the specified theoretical value of maximum water recovery ratio was not reached throughout the experiments, because the melt circulation pump would be exposed to a high risk of damage as the collecting tank would be almost empty for such operating conditions. In general, the water recovery results give a clear indication that the pilot plant, involving a single freezing stage, was capable of concentrating the RO brine and simultaneously producing a substantial amount of final product water of 
near seawater concentration, which can be recycled to join the feed stream and then easily desalted through a RO desalination plant.

Fig. 10d shows plots of the end-point cooling HTM temperature versus the actual operating time of the crystallisation process. As expected, the running time of the crystallisation process can be substantially reduced by decreasing the end-point cooling HTM temperature and cooling rate. This means that the production rate can be substantially increased by the end-point cooling HTM temperature and/or cooling rate.

Within the studied domain, the results of average growth rates were also considered and analytically computed, by assuming the dimensions and shape of solid layer ice to be cylindrical. The average growth rate is controlled by the crystallisation temperature and cooling rate. Fig. 10e shows the variation of product water salt concentration, as a function of the average growth rate. The average growth rate also has a strong influence on the quality of product water, in terms of salinity, where this parameter was found to be effective in removing significant amounts of dissolved salt from RO brine over a single stage of layer growth. For instance, the salt concentrations of product water were reduced from 4.44 wt $\%$ to $2.65 \mathrm{wt} \%$ when the average growth rate decreased from $0.22 \mathrm{~mm} / \mathrm{h}$ to $0.05 \mathrm{~mm} / \mathrm{h}$. These results showed that the adopted process is sensitive to variations in growth rate. However, there was no significant change in the salt concentration of product water observed, as the average growth rate varied from 0.22 to $0.47 \mathrm{~mm} / \mathrm{h}$, as shown in Fig.10e.

In the second series of experiments, the influence of feed concentration on the performance of the adopted technology was investigated. These experiments were carried out in a feed stage process with four different salt concentrations of $\mathrm{RO}$ brine ranging from $2.6 \mathrm{wt} \%$ to $6.1 \mathrm{wt} \%$. The crystallisation experiments were carried out at maximum water recovery ratio, $\mathrm{HTM}$ temperature of $-16^{\circ} \mathrm{C}$ and cooling rate of $0^{\circ} \mathrm{C} / \mathrm{min}$ respectively. The actual operating period of the crystallisation process in the experiments varied from 0.53 to $1.33 \mathrm{~h}$. A summary of the experimental results is given in Fig. 10f which shows the influence of feed concentration on the quality of product water, in terms of salt concentration. The general trend observed here shows agreement a previous study [5]. The quality of product water, in terms of salinity, is proportional to the feed salt concentration as shown in Fig. 10f. The lower feed concentration can significantly decrease the salt concentration of product water, resulting in improvement in the separation performance of the pilot plant. For instance, Fig. 10f shows that the salt concentrations of feed samples fed individually to the tested pilot plant are $2.6 \%, 3.4 \%, 5.1 \%$ and $6.1 \%$. The results showed that the FFFC process was capable of reducing the salt concentrations of product water down to an average of $1.84 \%, 2.3 \%, 3.4 \%$ and $4.45 \%$, for the same feed samples respectively.

The third series of the crystallisation experiments were carried out at end-point crystallisation temperatures of -10 and $-20^{\circ} \mathrm{C}$, whereas the cooling rates for these tests were set at a constant value of $0{ }^{\circ} \mathrm{C} / \mathrm{min}$. These tests were performed to detect and monitor the behaviour of the salt concentration of the product water for different crystallisation times. For the tests with an end-point crystallisation temperature of $-10^{\circ} \mathrm{C}$, five tests were conducted at various crystallisation times, which varied from 22.58 to 115.53 minutes. For the tests with an end-point crystallisation temperature of $-20^{\circ} \mathrm{C}$, five experiments were carried out at various crystallisation times ranging from 5 to 27.10 minutes. Figures $10 \mathrm{~g}$ and $10 \mathrm{~h}$ show a comparison between the salt rejection results, versus crystallisation time. For the tests with HTM temperatures of $-10^{\circ} \mathrm{C}$, the salt concentration of product water varied according to the crystallisation time. The maximum and minimum salt concentration values of product water were $3.63 \mathrm{wt} \%$ and $2.95 \mathrm{wt} \%$ respectively. These results were achieved at crystallisation times of 22.6 and 82.4 min respectively, as shown in Fig. 10g. For the tests with HTM temperatures of $-20^{\circ} \mathrm{C}$, the salt concentration of product water varied slightly according to the crystallisation time, where the maximum and minimum salt concentration 
values of product water were $4.29 \mathrm{wt} \%$ and $3.83 \mathrm{wt} \%$ respectively. These results were obtained at crystallisation times of 5.0 and 4.3 min respectively, as shown in Fig. 10h. By comparing Figures $10 \mathrm{~g}$ to $10 \mathrm{~h}$, a clear tendency of decreasing product water salt concentration with lower end-point crystallisation temperature can be observed, which confirmed the previous observations on the influence of crystallisation temperature on the product quality presented in Fig. 10a. Furthermore, the trend in the results for salt concentration of the product water versus the average growth rate is in agreement with the experimental data presented in Fig. 10e.

For the design of commercial plants, the recommended crystallisation temperatures range from $-12^{\circ} \mathrm{C}$ to $-16^{\circ} \mathrm{C}$ when the cooling rate is set to $0^{\circ} \mathrm{C} / \mathrm{min}$, whereas the recommended crystallisation temperature should not be higher than $-12^{\circ} \mathrm{C}$ when the cooling rate is set to below $0^{\circ} \mathrm{C} / \mathrm{min}$. Commercial plants with higher crystallisation temperatures (i.e. $>-12^{\circ} \mathrm{C}$ ) would require careful operation, and close monitoring to nucleate and form ice crystals that can be adhered to the crystalliser at the beginning of the crystallisation operation. Otherwise, several limitations might be encountered leading to an ice suspension forming inside the collecting tank, while plants with lower operating temperatures (i.e. < $16^{\circ} \mathrm{C}$ ) would be expected to be limited by operational cost.

\subsection{Multi-stage process (feed and rectification stages) without the sweating process}

In the fourth series of experiments, the influence of a multi-stage process, using the Sulzer FFFC process, on the degree of water desalination, in terms of salt rejection, water recovery, and ionic rejection, was investigated. A feed sample of RO brine was treated using two successive freezing stages, namely feed and rectification stages. The experiments were carried out at the maximum water recovery ratio. The crystallisation temperature and ramp of the feed and rectification stages were set to $-16^{\circ} \mathrm{C}$ and $0{ }^{\circ} \mathrm{C} / \mathrm{min}$ respectively. The actual operational periods of the feed and rectification stages were $0.83 \mathrm{~h}$ and $0.37 \mathrm{~h}$ respectively. A summary of the experimental and water chemistry data for the water samples of each freezing stage is given in Tables $2-4$.

Table 2 shows that the feed and rectification stages were able to lower the TDS value of the RO brine from 61,104 ppm to $32,451 \mathrm{ppm}$. Table 2 shows that the TDS value of the product water from each stage was 44,512 and $32,451 \mathrm{ppm}$ respectively. Thus the residual liquids, which were rejected from the feed and rectification stages, were concentrated with average TDS values of 147,840 and $95,258 \mathrm{ppm}$, respectively. The salt rejection ratios for the feed and rectification stages were 27.2 and $27.1 \%$, respectively, whilst the water recovery ratios were 84.17 and $76.3 \%$, for the same freezing stages respectively. Table 3 shows that the overall salt rejection and product water recovery ratios were $46.89 \%$ and $64.24 \%$, respectively. Table 4 shows the ionic composition of water samples of all the streams in the process. The results showed that the multi-stage process using the crystallisation process did not produce a final product reaching brackish water standards. Therefore, a post-treatment crystallisation process must be considered, when a high purity product water is required. 


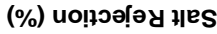

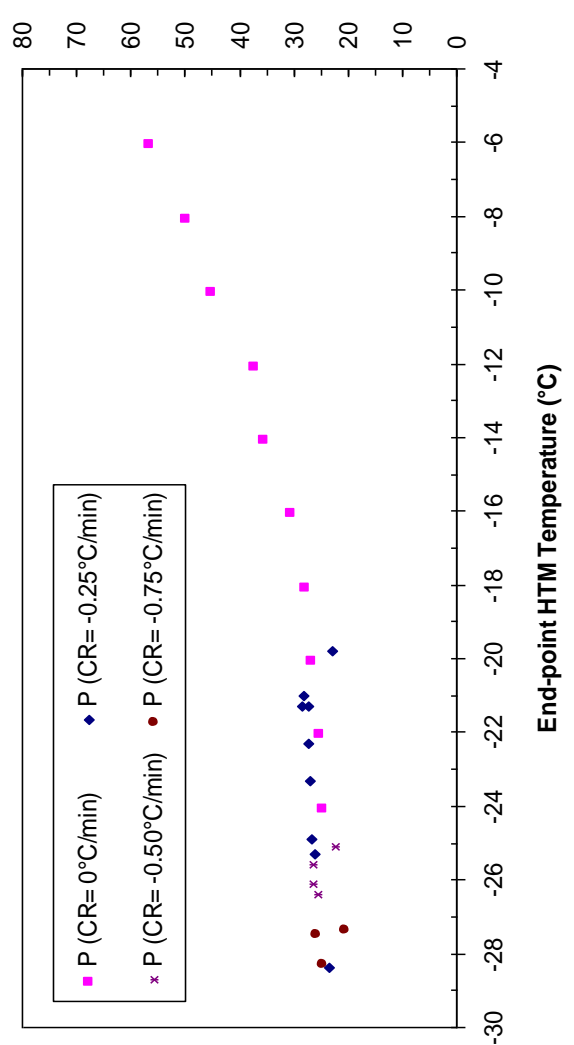

(\%łм) uo!̣eגłuəouoว ңןes

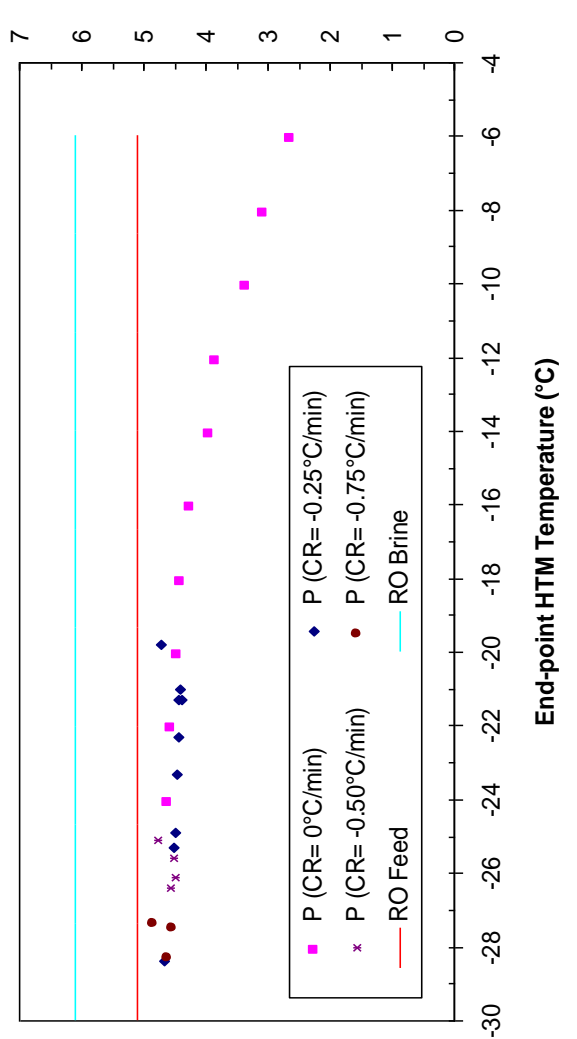

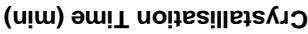

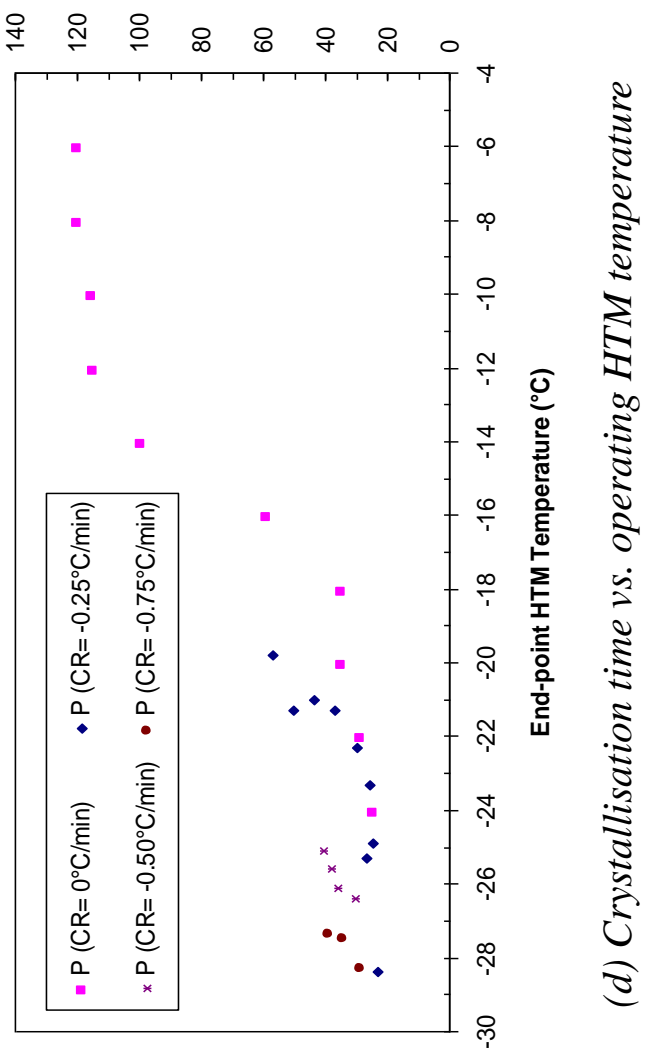

(\%) Кмәлоэәу мәјем

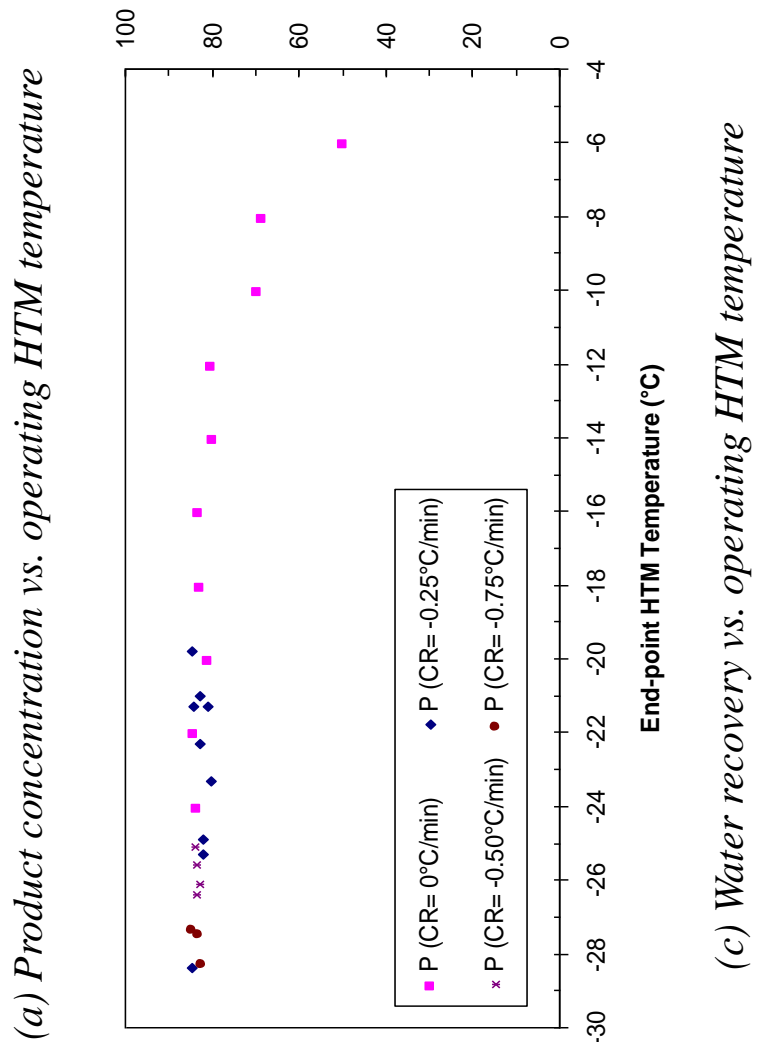



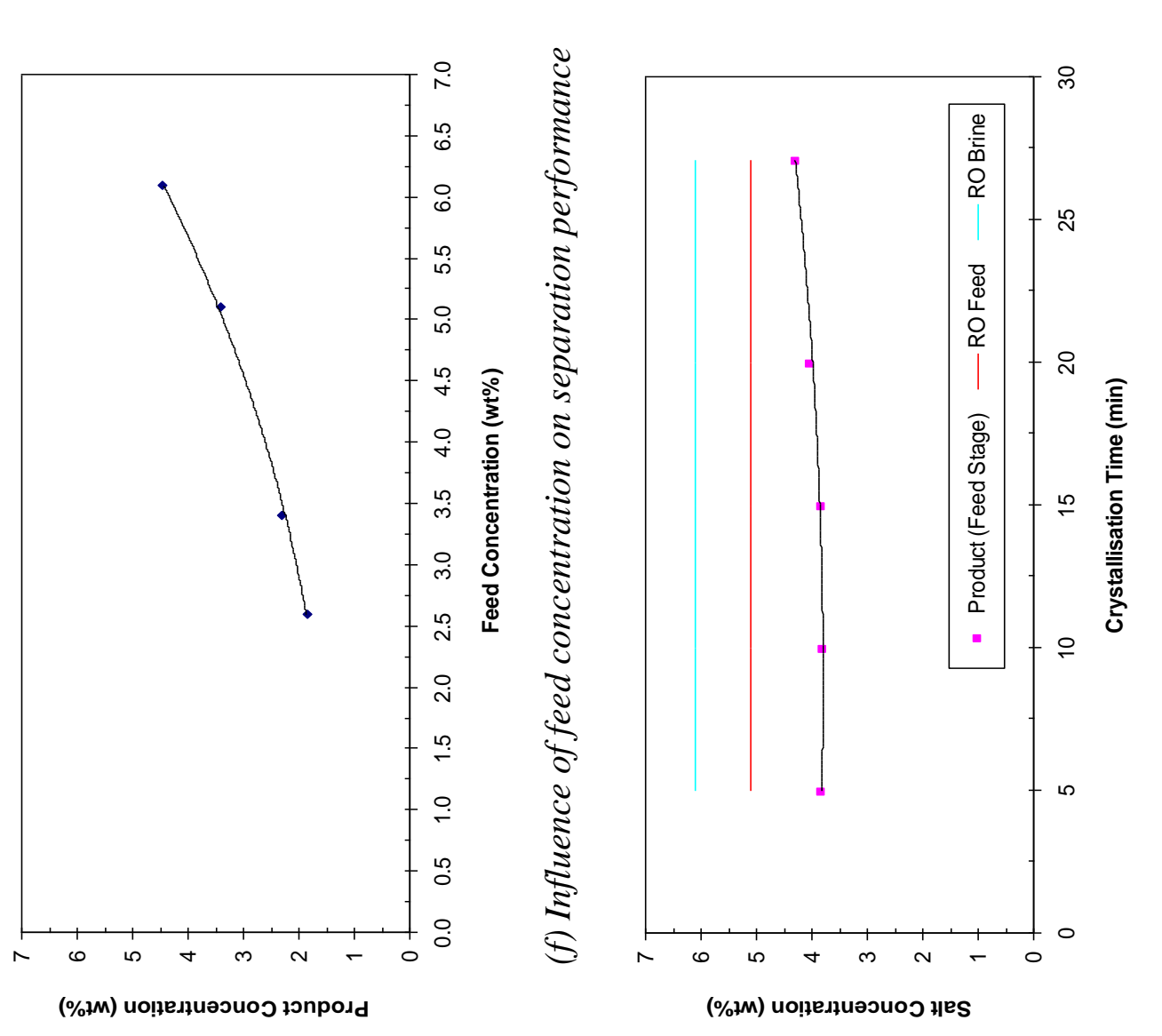

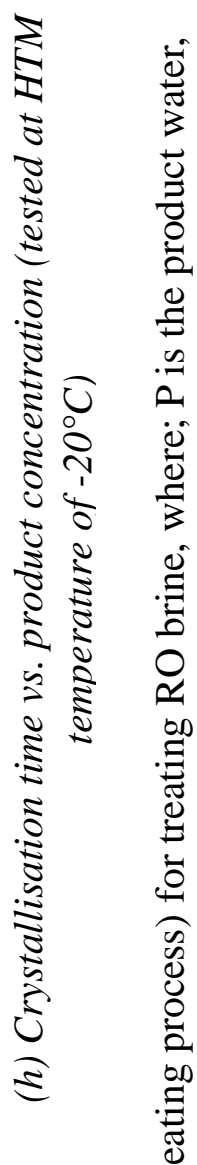
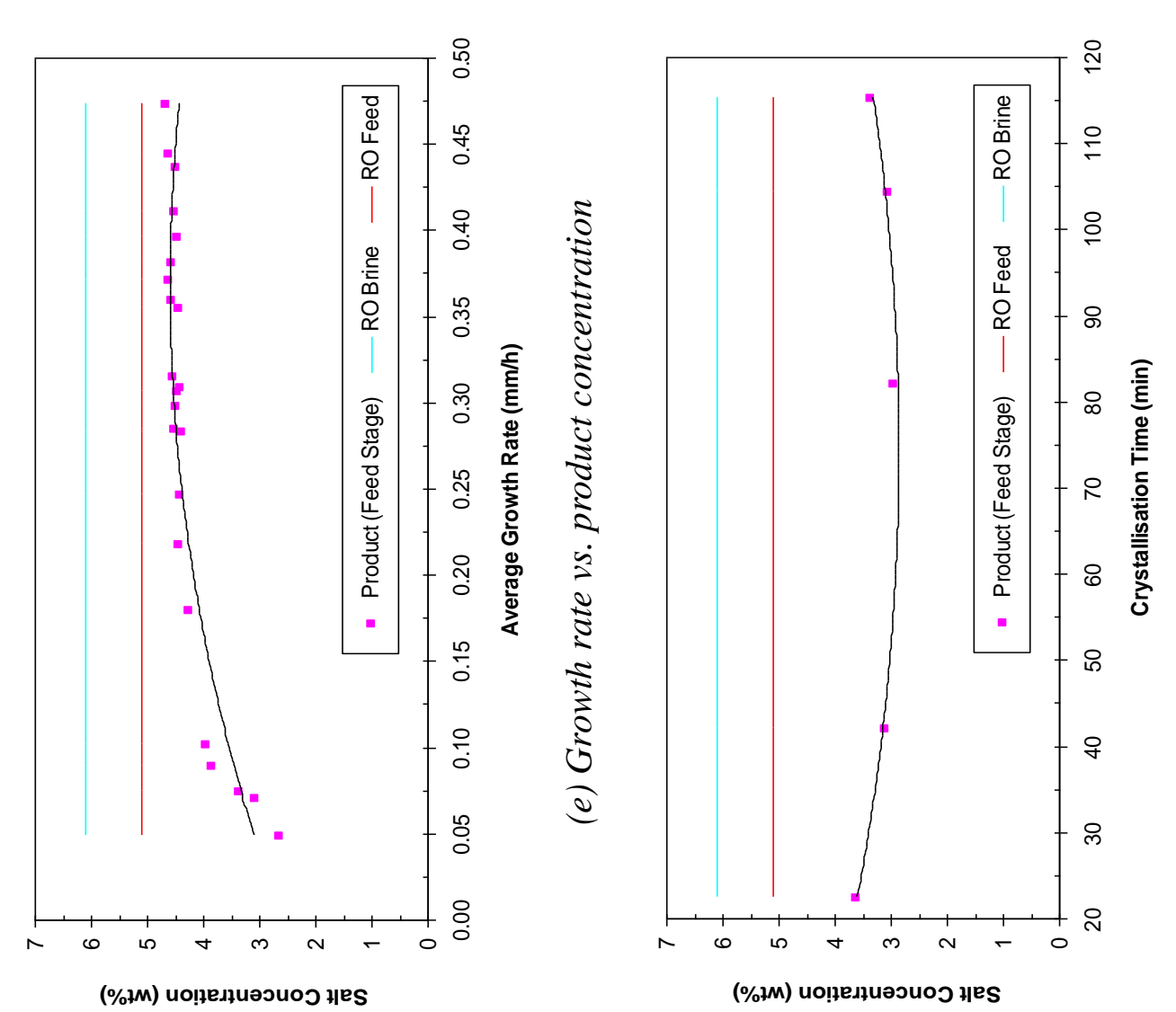

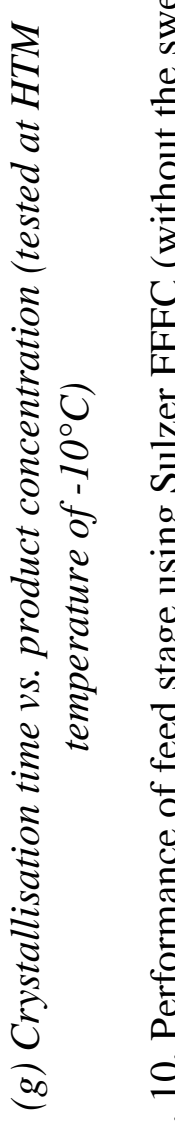

is 
Table 2

Summary of the performance data for the feed and rectification stages

\begin{tabular}{|c|c|c|c|c|}
\hline Stage & Water Sample & Parameters & Unit & Value \\
\hline \multirow{6}{*}{ Feed Stage } & \multirow{2}{*}{ Feed Water } & Mass & $\mathrm{kg}$ & 29.174 \\
\hline & & TDS & ppm & 61,104 \\
\hline & \multirow{2}{*}{ Product Water } & Mass & $\mathrm{kg}$ & 24.555 \\
\hline & & TDS & $\mathrm{ppm}$ & 44,512 \\
\hline & \multirow{2}{*}{ Residual Liquid } & Mass & $\mathrm{kg}$ & 4.619 \\
\hline & & TDS & $\mathrm{ppm}$ & 147,840 \\
\hline \multirow{6}{*}{ Rectification Stage } & \multirow{2}{*}{ Feed Water } & Mass & $\mathrm{kg}$ & 24.555 \\
\hline & & TDS & $\mathrm{ppm}$ & 44,512 \\
\hline & \multirow{2}{*}{ Product Water } & Mass & $\mathrm{kg}$ & 18.740 \\
\hline & & TDS & $\mathrm{ppm}$ & 32,451 \\
\hline & \multirow{2}{*}{ Residual Liquid } & Mass & $\mathrm{kg}$ & 5.815 \\
\hline & & TDS & ppm & 95,258 \\
\hline
\end{tabular}

Table 3

Overall data for the performance data for the feed and rectification stages

\begin{tabular}{lllll}
\hline Stage & Water Sample & Parameters & Unit & Value \\
\hline \multirow{3}{*}{ Overall } & \multirow{2}{*}{ Feed Water } & Mass & $\mathrm{kg}$ & 29.174 \\
& & TDS & $\mathrm{ppm}$ & 61,104 \\
\cline { 2 - 5 } & \multirow{2}{*}{ Product Water } & Mass & $\mathrm{kg}$ & 18.740 \\
& \multirow{2}{*}{ TDS } & $\mathrm{ppm}$ & 32,451 \\
\cline { 2 - 5 } & \multirow{2}{*}{ Residual Liquid } & Mass & $\mathrm{kg}$ & 10.434 \\
& & TDS & $\mathrm{ppm}$ & 112,566 \\
\hline
\end{tabular}

Table 4

Major physiochemical analysis of water samples for the feed and rectification stages without use of a sweating process for treating RO brine

\begin{tabular}{lllllllll}
\hline Parameter & Unit & \multicolumn{3}{c}{ Feed Stage } & \multicolumn{3}{c}{ Rectification Stage } & Total \\
& & Feed & Product & Residue & Feed & Product & Residue & Residue* \\
\hline $\mathrm{pH}$ & - & 7.41 & 7.18 & 7.24 & 7.18 & 7.16 & 7.19 & $7.19-7.24$ \\
$\mathrm{TDS}$ & $\mathrm{mg} / \mathrm{L}$ & 61,104 & 44,512 & 147,840 & 44,512 & 32,451 & 95,258 & 112,566 \\
Conductivity & $\mathrm{mS} / \mathrm{cm}$ & 76.4 & 55.1 & 165.1 & 55.1 & 39.5 & 117.1 & 134.8 \\
$\mathrm{Ca}^{2+}$ & $\mathrm{mg} / \mathrm{L}$ & 1,476 & 920 & 4,760 & 920 & 656 & 2,112 & 2,948 \\
$\mathrm{Mg}^{2+}$ & $\mathrm{mg} / \mathrm{L}$ & 1,463 & 877 & 3,717 & 877 & 419 & 3,051 & 2,860 \\
$\mathrm{Na}^{+}$ & $\mathrm{mg} / \mathrm{L}$ & 20,881 & 15,628 & 56,481 & 15,628 & 12,126 & 34,174 & 38,602 \\
$\mathrm{Cl}^{-}$ & $\mathrm{mg} / \mathrm{L}$ & 32,200 & 24,100 & 87,100 & 24,100 & 18,700 & 52,700 & 59,529 \\
$\left(\mathrm{SO}_{4}\right)^{2-}$ & $\mathrm{mg} / \mathrm{L}$ & 4,800 & 3,200 & 12,000 & 3,200 & 2,360 & 8,900 & 8,854 \\
$\mathrm{NO}_{3}^{-}$ & $\mathrm{mg} / \mathrm{L}$ & 2.0 & 1.7 & 3.3 & 1.7 & 1.1 & 4.6 & 3.3 \\
$\left(\mathrm{HCO}_{3}\right)^{-}$ & $\mathrm{mg} / \mathrm{L}$ as & 241.2 & 170.3 & 450.2 & 170.3 & 168.2 & 300.9 & 319.0 \\
\hline
\end{tabular}

\subsection{Crystallisation and sweating experiments using feed stage}

In the fifth group of experiments, the potential of the adopted technology, using crystallisation and sweating processes, was investigated for desalting and concentrating RO brine. These experiments were carried out in a feed stage process, and were performed at the maximum water recovery ratio. The tested salt concentrations of feed ranged from 2.6 to 6.1 $\mathrm{wt} \%$. The crystallisation temperature and ramp for the experiments were $-16^{\circ} \mathrm{C}$ and $0{ }^{\circ} \mathrm{C} / \mathrm{min}$ 
respectively, whereas the sweating experiments were carried out using a start-point HTM temperature of $-16^{\circ} \mathrm{C}$ and sweating ramp of $0.5^{\circ} \mathrm{C} / \mathrm{min}$. For clarity, the sweating time is identified and referred to, in the course of this paper, as time elapsed from increasing the temperature of the crystalliser until the end of collecting the last sample of sweating fractions. The crystal mass ratio is analytically computed by dividing the extracted mass of sweating fraction to the original mass of the product water, i.e. the mass of product before performing the sweating process.

In Figures 11a and $11 \mathrm{~b}$ the influence of salt concentration of feed water on the quality of product water is shown. The crystalline impurities were found to increase with increasing feed concentration, and to decrease with increasing crystal mass ratio and sweating times. For instance, for desalting the RO brine, the concentration of crystals was reduced from 4.31 wt $\%$ to $3.32 \mathrm{wt} \%$ after carrying out the sweating process at a mass crystal ratio of $17.19 \%$ and sweating time of $26.40 \mathrm{~min}$. In the case of desalting AG seawater, Figures 11a and 11b show that the salt concentration of crystals was reduced from $3.33 \mathrm{wt} \%$ to $2.64 \mathrm{wt} \%$ after carrying out the sweating process at a mass crystal ratio and sweating time of $15 \%$ and 24.13 min respectively.

In general, the variation in the behaviour of the product concentration versus the mass crystal ratio represents a reflection of the sweating efficiency. At the beginning of sweating operation where the first sweat fraction was taken, a dramatic fall in the product concentration can be observed as shown in Figures 11a and 11b. This was due to the remaining impure melt, adhered to the surface of the crystal layer, being removed efficiently by the initial sweating step. As the sweating continued, a slight decrease in the product concentration was observed, because the impurities were further inside the crystalline slab, and effectively trapped and occluded. Therefore, further sweating would be a progressive melting process for the remaining crystalline mass, which corresponds to a poor sweating efficiency. This procedure must also take into account that the product lost (i.e. mass crystal ratio) should be within an average of $10 \%$ [7]. Although the sweating time can be varied from $10 \mathrm{~min}$ to $30 \mathrm{~h}$, the sweating operation should be performed at the shortest retention time possible (about one-third of a crystallisation process); otherwise, the production rate will dramatically decline [7]. The production rate is a crucial factor for desalination applications. The production rate will be negatively affected by the sweating process because of the product loss, and an increase in the time retention of the freezing stage. From an economic point of view, the reduced amount of product water due to the sweating step for the rise in purity was not worthwhile for the purpose of desalination, as the pilot plant with sweating process did not produce a final product water suitable for human consumption.

For the cases of operating the pilot plant without sweating, the HTM temperature and cooling rate of the partial melting process were usually set to $15{ }^{\circ} \mathrm{C}$ and $0{ }^{\circ} \mathrm{C} / \mathrm{min}$. This procedure was applied to accelerate the process of partially melting the crystalline mass in order to recover enough product water, which enables the user to run the pump during the total melting process, to yield the final product water. These operating conditions were also considered in the subsequent sweating experiments, in order to carry out the sweating process in the fastest possible way, and also determine the trend for crystalline impurity, as a function of the crystal mass ratio and sweating times under these operating conditions.

Therefore, the sixth group of experiments was carried out with feed samples using $\mathrm{RO}$ brine to investigate the effect of the sweating rate on the salt concentration of product water. The experiments were carried out at maximum water recovery ratio. These experiments were also performed in a feed stage process, which was examined at crystallisation temperatures of -10 and $-20^{\circ} \mathrm{C}$. The crystalline impurity content was measured at different sweating rates ranging from 0.5 to $4.6{ }^{\circ} \mathrm{C} / \mathrm{min}$. 
Figures $11 \mathrm{c}$ and $11 \mathrm{~d}$ show the variation of product water salt concentration, as a function of the crystal mass ratio and sweating time at different crystallisation temperatures and sweating rates. The salt concentration of product water increases with decreasing crystallisation temperature, as observed previously in different experiments. For the experiments with a crystallisation temperature of $-20^{\circ} \mathrm{C}$ and sweating rate of $0.5{ }^{\circ} \mathrm{C} / \mathrm{min}$, the salt concentration of product water was reduced from 4.6 to $3.27 \mathrm{wt} \%$ when the crystal mass ratio increased from $0 \%$ to $17.85 \%$ (see Fig. 11c). For the experiments with a crystallisation temperature of $-20{ }^{\circ} \mathrm{C}$ and sweating rate of $4.6{ }^{\circ} \mathrm{C} / \mathrm{min}$, the salt concentration of product water was reduced from 4.6 to $3.83 \mathrm{wt} \%$, when the crystal mass ratio increased from $0 \%$ to $8.60 \%$ (see Fig. 11c). When the crystal mass ratio reached $8.60 \%$, the sweating process could not be continued, because the remaining crystal mass inside the crystalliser tube was dislodged, and fell into the collecting tank. This experiment was repeated several times and the same behaviour was observed, indicating that the maximum crystal mass ratio could not exceed $8.60 \%$. For the experiments with a crystallisation temperature of $-10{ }^{\circ} \mathrm{C}$ and sweating rate of $0.5^{\circ} \mathrm{C} / \mathrm{min}$, the salt concentration of product water was reduced from 2.89 to 1.86 wt $\%$, when the crystal mass ratio increased from $0 \%$ to $24.41 \%$ (see Fig. 11c). For the experiments with a crystallisation temperature of $-10{ }^{\circ} \mathrm{C}$ and sweating rate of $3.9{ }^{\circ} \mathrm{C} / \mathrm{min}$, the salt concentration of product water was deceased from $2.89 \mathrm{wt} \%$ to $2.38 \mathrm{wt} \%$, when the crystal mass ratio varied from $0 \%$ to $27.50 \%$ (see Fig. 11c). The remaining crystal mass was not dislodged from the crystalliser tube as seen in the previous experiments at $-20{ }^{\circ} \mathrm{C}$. The experimental results proved that lowering sweating rate can slightly reduce the product concentration. This gives clear evidence that the performance of the adopted technology cannot produce a final product of near drinking water at a reasonable production rate. Fig. $11 \mathrm{~d}$ shows that the sweating time was significantly reduced by increasing the sweating rate. For instance, the sweating time was reduced from $21.73 \mathrm{~min}$ to $7.62 \mathrm{~min}$ when the sweating rate was changed from 0.5 to $4.6^{\circ} \mathrm{C} / \mathrm{min}$. From an economic point of view, the product concentration was not significantly changed by changing the sweating rate, indicating that the user can carry out the sweating process in the fastest possible way to reduce the time retention of the freezing stage, if the sweating process is essential for such an application.

\subsection{Multi-stage processing (feed, rectification, and stripping stages) with a sweating procedure for treating $\mathrm{RO}$ brine}

The purpose of the seventh series of experiments was to evaluate the performance of a multistage process, using FFFC and sweating processes, for desalting and concentrating RO brine. The multi-stage process consisted of three successive freezing stages, i.e. feed, rectification, and stripping stages, and was operated at the maximum water recovery ratio. The experiments determined the performance of each freezing stage, with respect to the salt concentration of product water and ionic rejection. The crystallisation temperature and ramp for the feed and rectification stages were $-16^{\circ} \mathrm{C}$ and $0{ }^{\circ} \mathrm{C} / \mathrm{min}$ respectively, whereas the sweating steps were carried out at a start-point HTM temperature and sweating ramp of $16^{\circ} \mathrm{C}$ and $0.5^{\circ} \mathrm{C} / \mathrm{min}$ respectively. The stripping stage was initially tested with a crystallisation temperature of $-22^{\circ} \mathrm{C}$; however, the test was terminated at the beginning of the crystallisation operation, because of the collapse of the nucleated ice from the crystalliser tube, which led to a substantial amount of undesired ice suspension inside the collecting tank. This is because the initial crystal layer adhering like an encrustation on the cooled surfaces of the crystalliser tends to be porous, with a sponge-like structure, since the crystal layer was contained substantial amounts of impurities. This type of crystal layer tends to be weak in structure and does not stick well to the cooled surface of crystalliser, and cannot resist the incoming flow of circulated melt, which flows as a falling film. To prevent this problem, the stripping stage requires a well-controlled driving force and flow rate. 


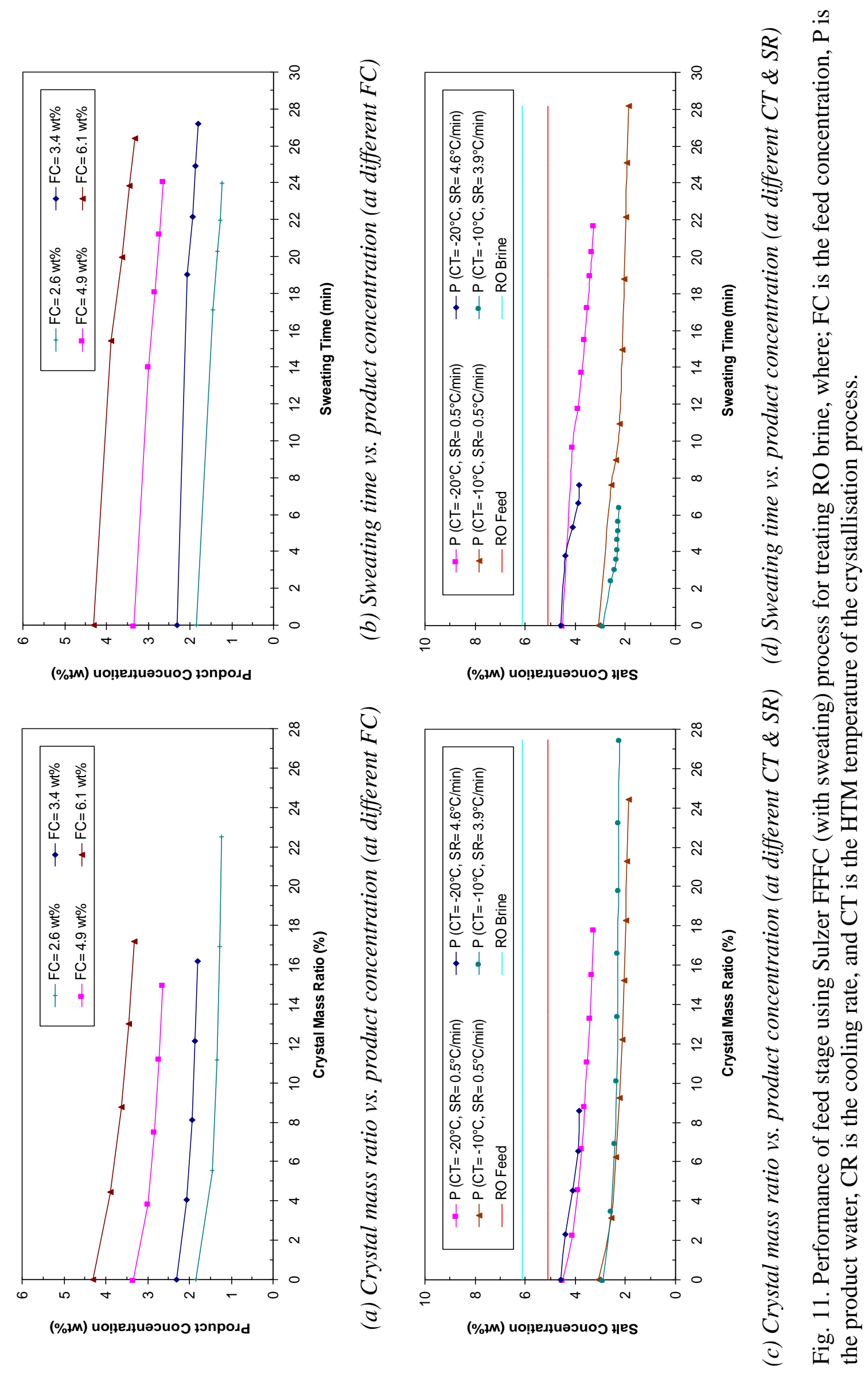


Therefore, the experiment for the stripping stage was carried out with the crystallisation temperature and ramp at $-26^{\circ} \mathrm{C}$ and $0{ }^{\circ} \mathrm{C} / \mathrm{min}$ respectively; furthermore, the flow rate of melt circulation pump was reduced at the beginning of the crystallisation operation for $5 \mathrm{~min}$, and then increased to the optimum flow rate. In general, the crystallisation operation of the stripping stage needs intensive monitoring to avoid the appearance of an ice suspension inside the collecting tank. The sweating steps were carried out at a start-point HTM temperature of $-26^{\circ} \mathrm{C}$ and sweating ramp of $0.5^{\circ} \mathrm{C} / \mathrm{min}$. The actual operational period of the crystallisation process for the feed, rectification, and stripping stages were $0.87,0.75$, and $0.56 \mathrm{~h}$ respectively, whereas the actual operational periods of the sweating process were 0.6 , 0.45 , and $0.47 \mathrm{~h}$, for the same freezing stages respectively. The amount of feed used each time was approximately $30 \mathrm{~kg}$, so the experiments for the feed stage were individually repeated 6 times to collect the water samples for physiochemical analysis, and also to collect enough feed water for the rectification and stripping stages, which were performed only one time to produce the final product water.

A summary of the performance data for the multi-stage process and physiochemical analysis for the water samples of each freezing stage is given in Tables 5 to 7 . Furthermore, the salt concentration of product water, water recovery ratio, and salt rejection ratio for each freezing stage is shown in Fig. 12.

Table 5 shows that the average TDS value of the brine fed to the feed stage, rectification, and stripping stages were 61,104, 33,701, and 141,974 ppm, whereas these freezing stages were capable of lowering the feed waters down to $33,701,17,913$, and $113,787 \mathrm{ppm}$ respectively. The calculations of the salt rejection ratios give $44.84,46.85$, and $19.85 \%$, for the same freezing stages respectively. The overall results proved that the tested technology was capable of reducing the TDS value of RO brine from 61,104 ppm down to 17,913 ppm over two successive freezing stages (see Tables 5 and 6). The calculation of the overall salt rejection ratio for these stages was $70.68 \%$. The results clearly indicate that the salt rejection ratio was improved by adding one more freezing stage (i.e. rectification stage); as a result, the salt rejection ratio is proportional to the number of freezing stages. The calculations of the water recovery ratios were $69.85,71.79$, and $69.72 \%$, for the same freezing stages respectively. The water recovery ratios for the freezing stages were approximately the same, because the crystallisation processes for these freezing stages were performed at the same and maximum ratios. The calculation of the overall water recovery ratio gives $50.15 \%$ (see Table 6 ). The experimental results clearly indicate that the water recovery ratio declined from $69.85 \%$ down to $50.15 \%$ by adding a rectification stage. This indicates that the water recovery ratio is inversely proportional to the number of freezing stages.

Table 5 shows that the TDS values of the residual liquid of the feed, rectification and stripping stages were $124,576,73,886$ and 218,033 ppm respectively. The residual liquid of the rectification stage can be further treated and concentrated by recycling this waste stream back to join the main feed stream of the feed stage, whereas the residual liquids of the feed and stripping stage cannot be treated and concentrated through the stripping stage. Although the sweating process was conducted with considerable loss of final product, poor separation performance was observed in the stripping stage. This was due to the high salinity feed fed to the stripping stage, which led to a crystal layer tending to be porous, in a sponge-like structure, where the impurities were kept further inside the structure of the crystal layer and occluded. The performance of the stripping might be improved by using a higher crystallisation temperature and higher sweating rate with considerable mass loss in the final product water through the sweating operation. However, a moderately effective sweating process with a significant decrease in the production rate (due to considerable loss of final product water) is expected for such operating conditions. 
Table 5

Summary of the performance data for the feed, rectification, and stripping stages

\begin{tabular}{|c|c|c|c|c|}
\hline Stage & Water Sample & Parameters & Unit & Value \\
\hline \multirow{6}{*}{ Feed Stage } & \multirow{2}{*}{ Feed Water } & Mass & $(\mathrm{kg})$ & 30.931 \\
\hline & & TDS & $(\mathrm{ppm})$ & 61,104 \\
\hline & \multirow{2}{*}{ Product Water } & Mass & $(\mathrm{kg})$ & 21.604 \\
\hline & & TDS & $(\mathrm{ppm})$ & 33,701 \\
\hline & \multirow{2}{*}{ Residual Water* } & Mass & $(\mathrm{kg})$ & 9.327 \\
\hline & & TDS & $(\mathrm{ppm})$ & 124,576 \\
\hline \multirow{6}{*}{ Rectification Stage } & \multirow{2}{*}{ Feed Water } & Mass & $(\mathrm{kg})$ & $30.827^{(a)}$ \\
\hline & & TDS & $(\mathrm{ppm})$ & 33,701 \\
\hline & \multirow{2}{*}{ Product Water } & Mass & $(\mathrm{kg})$ & 22.132 \\
\hline & & TDS & $(\mathrm{ppm})$ & 17,913 \\
\hline & \multirow{2}{*}{ Residual Water* } & Mass & $(\mathrm{kg})$ & 8.695 \\
\hline & & TDS & $(\mathrm{ppm})$ & 73,886 \\
\hline \multirow{6}{*}{ Stripping Stage } & \multirow{2}{*}{ Feed Water } & Mass & $(\mathrm{kg})$ & $27.648^{(b)}$ \\
\hline & & TDS & $(\mathrm{ppm})$ & 141,974 \\
\hline & \multirow{2}{*}{ Product Water } & Mass & $(\mathrm{kg})$ & 19.276 \\
\hline & & TDS & $(\mathrm{ppm})$ & 113,787 \\
\hline & \multirow{2}{*}{ Residual Water* } & Mass & $(\mathrm{kg})$ & 8.372 \\
\hline & & TDS & $(\mathrm{ppm})$ & 218,033 \\
\hline
\end{tabular}

* Residual liquid includes the overall results of the actual residual liquid and sweat fractions.

(a) In order to run the stage a minimum volume is required in the feed tank (hence this is greater than the product water from the feed stage)

(b) In this case the feed water is slightly higher in concentration than the residual water as multiple runs were done on the feed stage to obtain enough residual water to process in the stripping stage.

As for the chemistry data of the water samples obtained from each freezing stage, Table 7 shows that the ionic composition of the major ions in each process stream. With regard to the purification efficiency of the sweating process, the salt concentrations of the product water were found to be proportional to the mass of sweat fraction as shown in Fig. 12. Figure 12 also shows the influence of crystal mass ratio on the salt rejection ratio, and water recovery ratio. The salt rejection ratio was found to be proportional to the crystal mass ratio, whereas the water recovery ratio was found to be inversely proportional to the crystal mass ratio.

Table 6

Overall data for the performance data for the feed, rectification, and stripping stages

\begin{tabular}{lllll}
\hline Stage & Water Sample & Parameters & Unit & Value \\
\hline \multirow{4}{*}{ Overall } & \multirow{2}{*}{ Feed Water } & Mass & $(\mathrm{kg})$ & 30.931 \\
& & TDS & $(\mathrm{ppm})$ & 61,104 \\
\cline { 2 - 5 } & \multirow{2}{*}{ Product Water } & Mass & $(\mathrm{kg})$ & 15.51 \\
& & TDS & $(\mathrm{ppm})$ & 17,913 \\
\cline { 2 - 5 } & \multirow{2}{*}{ Residual Liquid } & Mass & $(\mathrm{kg})$ & 15.42 \\
& & TDS & $(\mathrm{ppm})$ & 104,551 \\
\hline
\end{tabular}

By comparing the results of the fraction in each freezing stage (see Table 7), the salt and ionic concentrations of the fraction were deceased when compared to that in the previous fraction. For the feed and rectification stages, the impurity concentration in the last sweat fraction (i.e. fraction 4) is almost the same as the impurity concentration in the remainder of the crystal mass i.e. product water (see Table 7). This means that, a further sweating process 
will be a waste of product and sweating time, since there is no significant difference between the salt concentration of product and last sweat fraction. In fact, a further sweating operation will only be a total melting process for the remaining crystal mass. The remaining impurities can be considered to be locked-in inside the crystal mass, which can be further purified by one of the following methods: (a) optimising the operating conditions of crystallisation and sweating operations; or (b) applying a multistage process. In contrast with the stripping stage (see Table 7), the sweating operation can proceed further since the impurity concentration of last sweat fraction (i.e. fraction 3) was still higher than that in the impurity concentration in the remainder of the crystal mass. This means that the sweating time needed to be extended further, in order to purify the remainder of the crystal mass, i.e. product water. However, the suggested application will significantly increase the overall time of the freezing stage, and decrease the overall water recovery ratio, which will eventually lead to a significant reduction in the production rate of the stripping stage. Thus, the stripping stage using the adopted technology was found not to be technically feasible for further concentrating the already concentrated solutions of RO brine. A static crystallisation process might be an alternative solution in such applications.

Based on visual observation, there was clear signs of solid salts precipitation in the remaining residual liquid, and in the pilot plant equipment while carrying out the experiment for the stripping stage. Table 5 demonstrates and confirms the signs of precipitation of salts composed of $\mathrm{Ca}^{2+}, \mathrm{Mg}^{2+}$, and $\left(\mathrm{SO}_{4}\right)^{2-}$ ions, since the calculations of the percentage losses in their ionic concentration are about 26,9 , and $14 \%$ respectively. The reason for the solid salts precipitation may be explained by one of the following; (i) the stripping stage was examined at very low crystallisation temperature, which was lower than the eutectic temperature of some salts in the residual liquid. Following van der Ham et al. [16], a summary of several eutectic points and eutectics of chemical composition for different binary salt-water systems are shown in Fig. 13. For example, sodium sulphate precipitated as $\mathrm{Na}_{2} \mathrm{SO}_{4} \cdot 10 \mathrm{H}_{2} \mathrm{O}$ at a eutectic composition of $12.7 \mathrm{wt} \%$ and eutectic temperature of $-3.6^{\circ} \mathrm{C}$; (ii) the solubility limit of some salts in the residue might have been reached. Table 8 shows the solubility limits of major salts available in seawater; and (iii) precipitation of solid sodium sulphate (i.e. $\mathrm{Na}_{2} \mathrm{SO}_{4} \cdot 10 \mathrm{H}_{2} \mathrm{O}$ ) in the residue, when the temperature of residue is reduced below $-8.2^{\circ} \mathrm{C}$ [17]. The solid salt phase begins to appear in the residue, although the eutectic temperature for the sodium sulphate salt is $-11^{\circ} \mathrm{C}$ [17].

The results proved that the sweating processes was able to lower the salt concentration of product water and ionic concentrations, and more specifically was found effective in feed and rectification stages (see Table 7 and Fig. 12). However, the main disadvantages of the sweating process are; reduction in water recovery ratio (see Fig. 12) and an increase in the running time of the freezing stage. On the other hand, the study proved that the purification efficiency of the sweating process was strongly dependant on the salt concentration of the original mass of crystal layer, as shown in Fig. 12. Figures $12 \mathrm{~g}$ and $12 \mathrm{~h}$ showed that the purification efficiency of sweating processes declined considerably on increasing the salt concentration of the original mass of the crystal layer. Although the results are quite encouraging in terms of the separation performance, the adopted technology, over two successive freezing stages, did not produce a final product close to potable water standards, with respect to salt concentration and ionic compositions, as illustrated in Table 7. However, the adopted technology produced a saline water of near brackish water standards that can be further easily desalted by: (a) optimising the crystallisation and sweating operations by compromising the purification efficiency and product loss; (b) increasing the number of rectification stages; and (c) using one of the conventional desalination technologies, such as brackish water RO membranes, to obtain a final product of drinking water. 
Table 7

Major physiochemical analysis of water samples for the feed and rectification stages with use of sweating process for treating RO brine

\begin{tabular}{|c|c|c|c|c|c|c|c|c|c|}
\hline \multirow[t]{3}{*}{ Parameter } & \multirow[t]{3}{*}{ Unit } & \multicolumn{7}{|c|}{ Feed Stage } & \multirow{3}{*}{$\begin{array}{l}\text { Total } \\
\text { Residue }\end{array}$} \\
\hline & & \multirow{2}{*}{ Feed } & \multirow{2}{*}{ Product } & \multirow{2}{*}{ Residue } & \multicolumn{4}{|c|}{ Fraction Fraction Fraction Fraction } & \\
\hline & & & & & 1 & 2 & 3 & 4 & \\
\hline$\overline{\mathrm{pH}}$ & - & 7.41 & 7.17 & 7.39 & 7.35 & 7.50 & 7.49 & 7.48 & $7.35-7.50$ \\
\hline TDS & $\mathrm{mg} / \mathrm{L}$ & 61,104 & 33,701 & 145,393 & 130,368 & 93,503 & 77,988 & 64,409 & 124,576 \\
\hline Conductivit & $\mathrm{y} \mathrm{mS} / \mathrm{cm}$ & 76.6 & 41.1 & 163.2 & 150.9 & 115.2 & 97.5 & 80.8 & 145.8 \\
\hline $\mathrm{Ca}^{2+}$ & $\mathrm{mg} / \mathrm{L}$ & 1,476 & 1,008 & 4,288 & 3,308 & 2,080 & 1,632 & 1,296 & 3,244 \\
\hline $\mathrm{Mg}^{2+}$ & $\mathrm{mg} / \mathrm{L}$ & 1,463 & 909 & 3,076 & 2,304 & 2,075 & 2,044 & 1,657 & 2,576 \\
\hline $\mathrm{Na}^{+}$ & $\mathrm{mg} / \mathrm{L}$ & 20,881 & 13,164 & 47,403 & 41,567 & 27,819 & 23,410 & 16,082 & 37,877 \\
\hline $\mathrm{Cl}^{-}$ & $\mathrm{mg} / \mathrm{L}$ & 32,200 & 20,300 & 73,100 & 64,100 & 42,900 & 36,100 & 24,800 & 58,410 \\
\hline$\left(\mathrm{SO}_{4}\right)^{2-}$ & $\mathrm{mg} / \mathrm{L}$ & 4,800 & 3,200 & 12,150 & 9,640 & 7,270 & 6,560 & 6,710 & 9,970 \\
\hline $\mathrm{NO}_{3}^{-}$ & $\mathrm{mg} / \mathrm{L}$ & 2.0 & 2.0 & 3.1 & 3.1 & 2.8 & 2.6 & 2.2 & 2.9 \\
\hline$\left(\mathrm{HCO}_{3}\right)^{-}$ & $\begin{array}{l}\mathrm{mg} / \mathrm{L} \text { as } \\
\mathrm{Ca} \mathrm{CO} \mathrm{CO}_{3}\end{array}$ & 241.2 & 153.4 & 498.6 & 452.4 & 312.8 & 256.4 & 201.2 & 407.6 \\
\hline
\end{tabular}

Table 7

Continued

\begin{tabular}{|c|c|c|c|c|c|c|c|c|c|}
\hline \multirow[t]{3}{*}{ Parameter } & \multirow[t]{3}{*}{ Unit } & \multicolumn{7}{|c|}{ Rectification Stage } & \multirow{3}{*}{$\begin{array}{l}\text { Total } \\
\text { Residue }\end{array}$} \\
\hline & & \multirow{2}{*}{ Feed } & \multirow{2}{*}{ Product } & \multirow{2}{*}{ Residue } & \multicolumn{4}{|c|}{ Fraction Fraction Fraction Fraction } & \\
\hline & & & & & 1 & 2 & 3 & 4 & \\
\hline $\mathrm{pH}$ & - & 7.17 & 7.00 & 7.20 & 7.25 & 7.28 & 7.20 & 7.19 & $7.00-7.28$ \\
\hline TDS & $\mathrm{mg} / \mathrm{L}$ & 33,701 & 17,913 & 97,880 & 73,917 & 46,814 & 38,202 & 31,746 & 73,886 \\
\hline Conductivit & $\mathrm{mS} / \mathrm{cm}$ & 41.1 & 21.4 & 119.9 & 92.6 & 58.1 & 46.9 & 38.6 & 92.4 \\
\hline $\mathrm{Ca}^{2+}$ & $\mathrm{mg} / \mathrm{L}$ & 1,008 & 498 & 2,160 & 1,640 & 1,040 & 800 & 636 & 1,605 \\
\hline $\mathrm{Mg}^{2+}$ & $\mathrm{mg} / \mathrm{L}$ & 909 & 521 & 2,109 & 2,070 & 928 & 753 & 682 & 1,618 \\
\hline $\mathrm{Na}^{+}$ & $\mathrm{mg} / \mathrm{L}$ & 13,164 & 8,041 & 32,423 & 30,543 & 16,795 & 14,007 & 13,034 & 25,638 \\
\hline $\mathrm{Cl}^{-}$ & $\mathrm{mg} / \mathrm{L}$ & 20,300 & 12,400 & 50,000 & 47,100 & 25,900 & 21,600 & 20,100 & 39,536 \\
\hline$\left(\mathrm{SO}_{4}\right)^{2-}$ & $\mathrm{mg} / \mathrm{L}$ & 3,200 & 1,260 & 7,000 & 6,000 & 3,200 & 3,000 & 1,580 & 5,254 \\
\hline $\mathrm{NO}_{3}^{-}$ & $\mathrm{mg} / \mathrm{L}$ & 2.0 & 1.1 & 2.8 & 2.3 & 2.1 & 1.9 & 1.6 & 2.4 \\
\hline$\left(\mathrm{HCO}_{3}\right)^{-}$ & $\begin{array}{l}\mathrm{mg} / \mathrm{L} \text { as } \\
\mathrm{CaCO} \mathrm{CO}_{3}\end{array}$ & 153.4 & 120.2 & 320 & 259.3 & 180.3 & 160.2 & 150.2 & 255 \\
\hline
\end{tabular}


Table 7

Continued

\begin{tabular}{|c|c|c|c|c|c|c|c|c|}
\hline \multirow[t]{3}{*}{ Parameter } & \multirow[t]{3}{*}{ Unit } & \multicolumn{6}{|c|}{ Stripping Stage } & \multirow{3}{*}{$\begin{array}{l}\text { Total } \\
\text { Residue }\end{array}$} \\
\hline & & \multirow[t]{2}{*}{ Feed } & \multirow[t]{2}{*}{ Product } & \multirow[t]{2}{*}{ Residue } & \multicolumn{3}{|c|}{ Fraction Fraction Fraction } & \\
\hline & & & & & 1 & 2 & 3 & \\
\hline $\mathrm{pH}$ & - & 7.39 & 7.6 & 7.15 & 7.20 & 7.28 & 7.40 & $7.15-7.40$ \\
\hline TDS & $\mathrm{mg} / \mathrm{L}$ & 145,393 & 113,787 & 217,482 & 212,038 & 198,966 & 177,876 & 218,033 \\
\hline Conductivit & $\mathrm{mS} / \mathrm{cm}$ & 163.2 & 135.9 & 210.0 & 207.0 & 199.5 & 186.4 & 210.3 \\
\hline $\mathrm{Ca}^{2+}$ & $\mathrm{mg} / \mathrm{L}$ & 4,288 & 3,230 & 4,716 & 4,660 & 4,456 & 4,436 & 4,632 \\
\hline $\mathrm{Mg}^{2+}$ & $\mathrm{mg} / \mathrm{L}$ & 3,076 & 1,021 & 1,419 & 1,389 & 1,300 & 1,264 & 1,376 \\
\hline $\mathrm{Na}^{+}$ & $\mathrm{mg} / \mathrm{L}$ & 47,403 & 38,908 & 78,464 & 72,628 & 64,846 & 56,416 & 72,648 \\
\hline $\mathrm{Cl}^{-}$ & $\mathrm{mg} / \mathrm{L}$ & 73,100 & 60,000 & 121,000 & 112,000 & 100,000 & 87,000 & 112,032 \\
\hline$\left(\mathrm{SO}_{4}\right)^{2-}$ & $\mathrm{mg} / \mathrm{L}$ & 12,150 & 9,360 & 15,900 & 16,120 & 14,780 & 13,800 & 1,484 \\
\hline $\mathrm{NO}_{3}{ }^{-}$ & $\mathrm{mg} / \mathrm{L}$ & 3.1 & 2.2 & 5.1 & 4.8 & 4.5 & 3.3 & 4.7 \\
\hline$\left(\mathrm{HCO}_{3}\right)^{-}$ & $\begin{array}{l}\mathrm{mg} / \mathrm{L} \text { as } \\
\mathrm{CaCO} \mathrm{CO}_{3}\end{array}$ & 498.6 & 350.3 & 520.2 & 500.3 & 497.5 & 460.6 & 505.9 \\
\hline
\end{tabular}




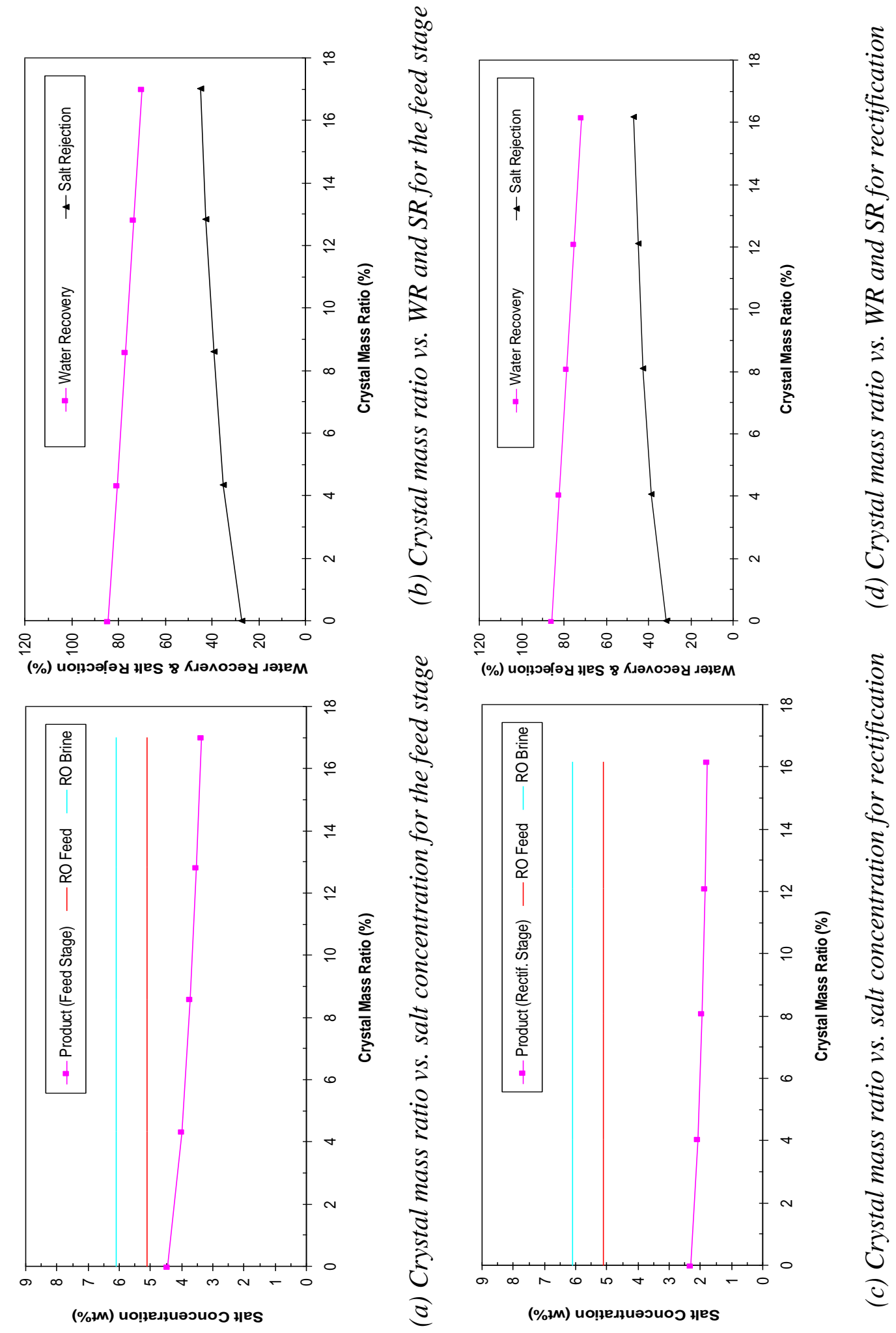



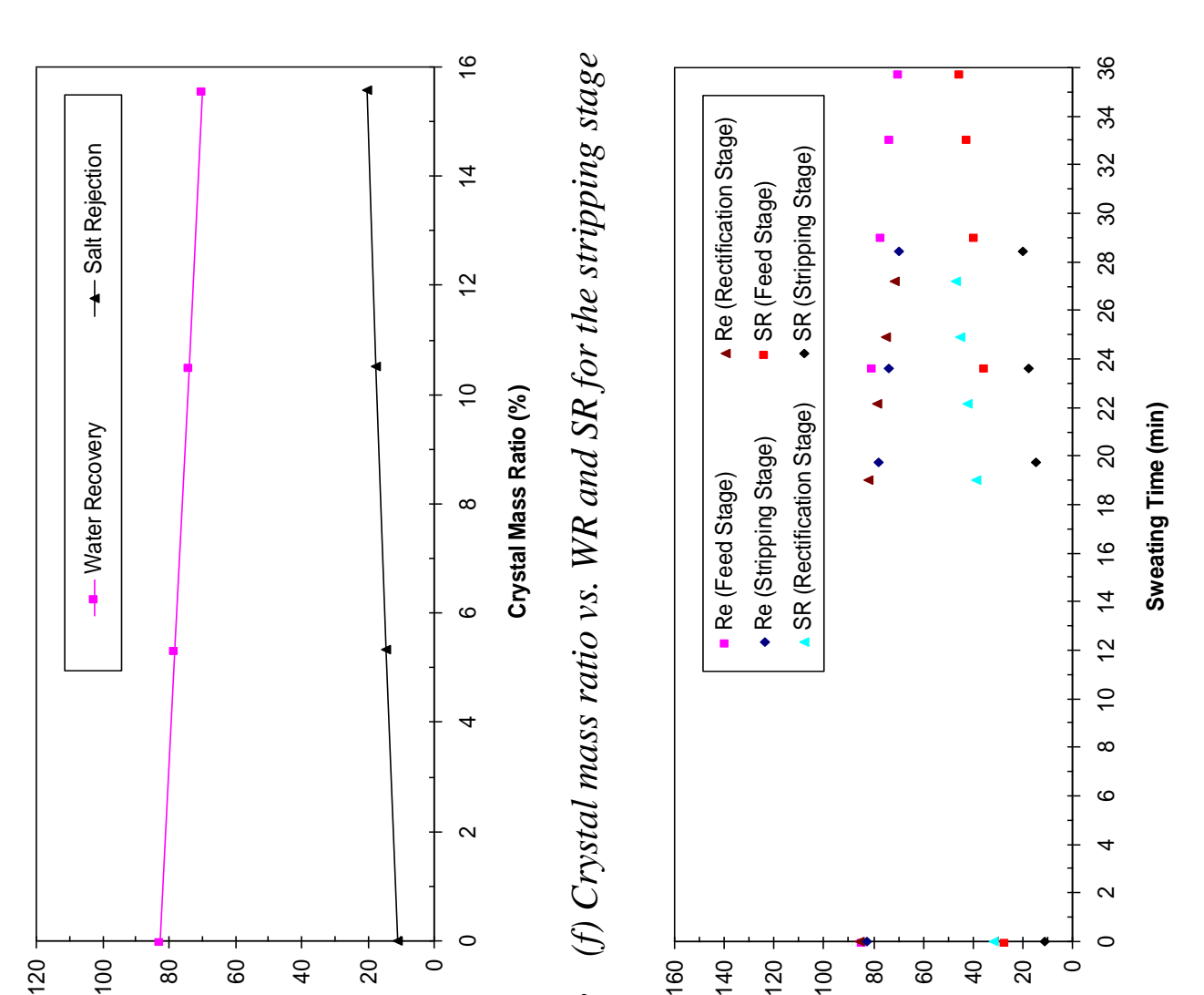

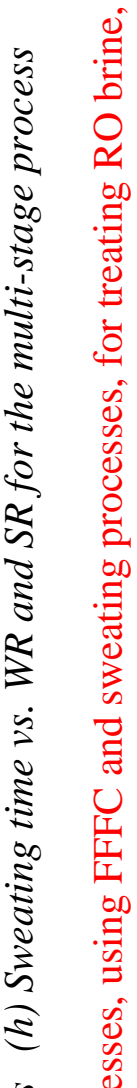

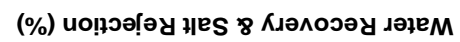

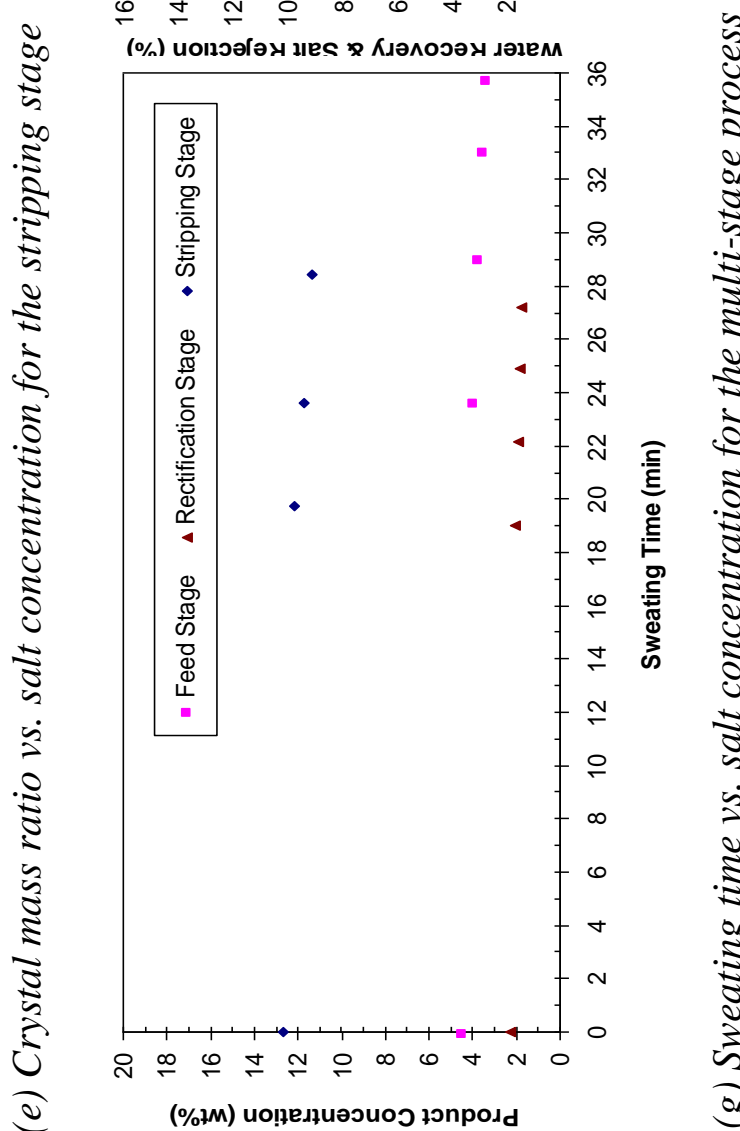

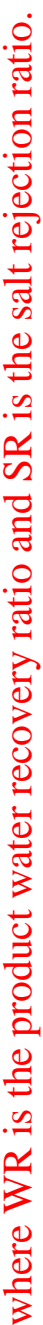

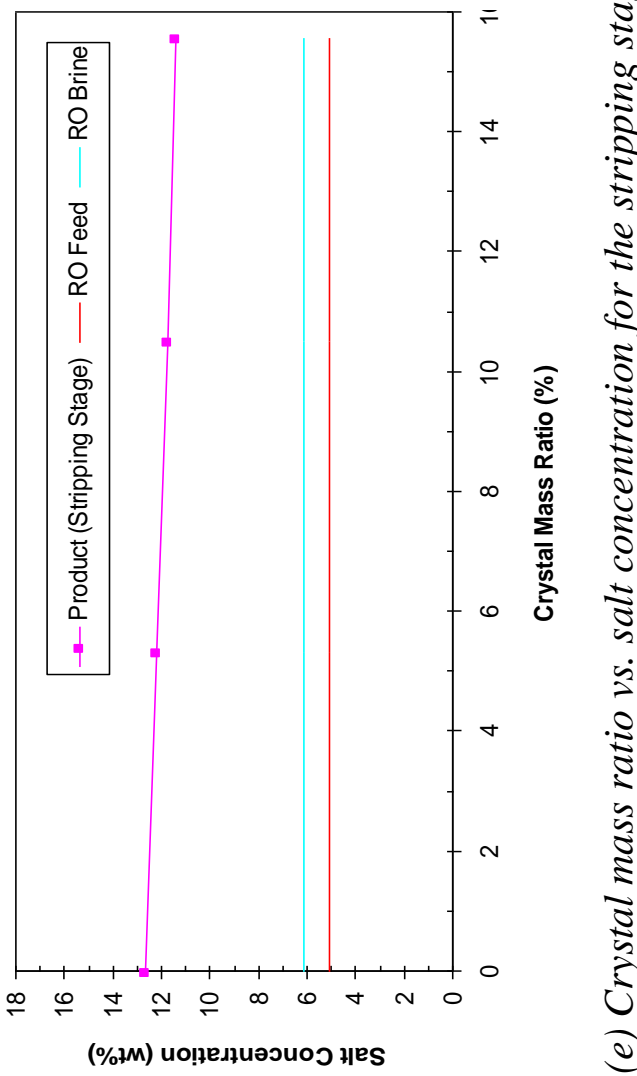

(\%łм) uo!̣eגłuəouos łonpoגd 


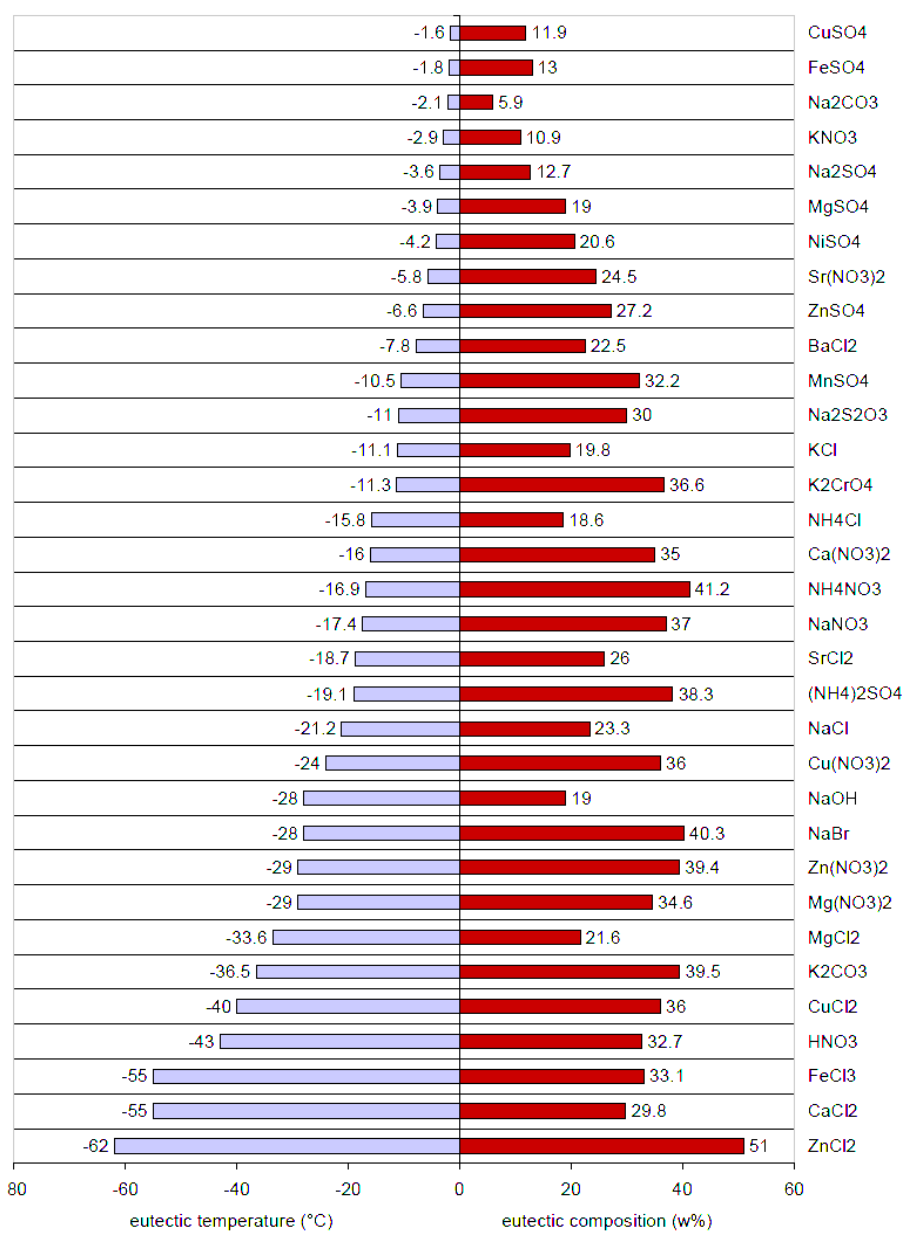

Fig. 13. Summary of several eutectic temperatures and chemical composition of binary saltwater systems [16].

Table 8

Results of measurements of solubility limits of major salts available in seawater, where subscript notations such as (f) and (s) mean feed and solution respectively

\begin{tabular}{lll}
\hline \multirow{2}{*}{ Salt } & Seawater Concentration* & Salt Concentration \\
\cline { 2 - 3 } & $\left(\mathrm{kg}_{(\mathrm{f})} / \mathrm{kg}_{(\mathrm{s})}\right)$ & $\left(\mathrm{mg} / \mathrm{kg}_{(\mathrm{s})}\right)$ \\
\hline $\mathrm{CaCO}$ & 1.9 & 370 \\
$\mathrm{Mg}(\mathrm{OH})_{2}$ & 1.9 & 440 \\
$\mathrm{CaSO}$ & 3.33 & 5,130 \\
$\mathrm{NaCl}$ & 8.9 & 278,000 \\
$\mathrm{KCl}$ & 10.5 & 9,030 \\
$\mathrm{MgCl}_{2}$ & 13.4 & 63,700 \\
\hline
\end{tabular}

* Seawater concentration is determined as a geometric progression of the mass ratios i.e. $\left(\mathrm{M}_{\text {(initial) }}\right) /\left(\left(\mathrm{M}_{\text {(initial) }}\right)-\left(\mathrm{M}_{\text {(deionised water })}\right)\right)$ [18].

\section{Conclusions}

In this study, the performance of FFFC and sweating processes, using Sulzer's FFFC pilot plant, was assessed and validated for concentrating RO retentate. Several parameters influencing the separation performance of crystallisation and sweating processes were investigated in a pilot plant operating in batch mode. Furthermore, a multistage process, with 
and without use of sweating process, was tested for RO brine. In general, the experimental results showed that feed concentration, crystallisation temperature, cooling and sweating rate, and average growth rate, had a significant influence on the separation performance of the treatment system. The Sulzer FFFC pilot plant, within a single freezing stage, and without use of a sweating process, was able to concentrate a significant amount of RO brine, while producing saline water to near seawater quality. In fact, the major physiochemical analysis of the product water showed that the product water was higher, in terms of quality, than typical RO plant feed water. This means that the product water was ready for immediate re-use as feed water to a RO desalination plant. The experimental results showed that the maximum water recovery ratio was $86 \%$. Therefore, the results for the RO brine experiments were highly encouraging for RO brine concentration. Based on these results, the investigated treatment system can be scaled up for such an application. As a result, a substantial annual rate of waste stream could be recycled and reused as RO feed, while preserving a large volume of natural water resource from exploitation, as well as minimising the waste stream as far as possible. On the other hand, the results for the stripping stage using the FFFC process were not encouraging. Although a sweating process was used, the stripping stage provided final product water with a salt concentration much higher than in RO brine.

\section{Acknowledgements}

The author would like to thank Sulzer Chemtech Ltd. for kindly supplying equipment and for their assistance during the pilot trials, particularly S. Dette, M. Stepanski, F. Lippuner and H. Engstler. Also, the author is thankful to the Director General of the Kuwait Institute for Scientific Research (KISR) and Executive Director of Water Research Centre, KISR for their support and encouragement towards this research.

\section{References}

1. P. Chelme-Ayala, D. W. Smith and M. G. El-Din, Membrane concentration management options: a comprehensive critical review, Can. J. Civ. Eng., 36 (6) (2009) 1107 - 1119.

2. M. Ahmad and P. Williams, Assessment of desalination technologies for high saline brine applications - Discussion Paper, Desalin.Water Treat., 30 (2011) 22-36.

3. P. Wang and T. Chung, A conceptual demonstration of freeze desalination - membrane distillation (FD-MD) hybrid desalination process utilizing liquefied natural gas (LNG) cold energy, Water Res., 46 (2012) 4037-4052.

4. J. Chang, J. Z. Kang-Jia Lu and T. Chung, Freeze desalination of seawater using LNG cold energy, Water Res., 102 (2016) 282-293.

5. P. Williams, M. Ahmad and B. Connolly, Freeze desalination: An assessment of an ice maker machine for desalting brines, Desalination, 308 (2013) 219-224.

6. P. M. Williams, M. Ahmad, B. S. Connolly and D. L. Oatley-Radcliffe, Technology for freeze concentration in the desalination industry, Desalination, 356 (2015) 314-327.

7. J. Ulrich, and H. Glade, Melt crystallisation, Shaker Verlag, ISBN: 3-8322-1533-6, Germany, 2003.

8. Sulzer, 2004. Suspension crystallisation technology, Sulzer Chemtech Ltd., Switzerland. [Online]http://www.sulzerchemtech.com/portaldata/11/Resources//Brochures/PT/Suspen sion_Crystallization_Technology.pdf $>$.

9. M. Ahmad, D. L. Oatley-Radcliffe, N. Hilal and P. M. Williams. Can a hybrid ROFreeze process lead to sustainable water supplies?, Desalination, 431 (2018) 140-150.

10. Sulzer Chemtech Ltd, 2011. Suspension crystallization. [Online] Available at: < http://www.sulzerchemtech.com/desktopdefault.aspx/tabid-2245>. 
11. A. Myerson, Handbook of industrial crystallisation, 2nd edition, ButterworthHeinemann, ISBN: 0750670126.

12. Sulzer, 2005. Sulzer Chemtech, Fractional crystallisation, Sulzer Chemtech Ltd., Switzerland. [Online] Available at: <http://www.sulzerchemtech.com/portaldata/11/Resources//Brochures/PT/Fractional_Cr ystallization-e.pdf>.

13. O. K. Buros, ABCs of desalting, $2^{\text {nd }}$ ed. S.I., International Desalination Association (IDA), Topsfield, Massachusetts, United States of America, 2000.

14. K. S. Spiegler and Y. M. El-Sayed, A desalination primer, Italy: Balaban Desalination Publications, ISBN: 0866890343, Rome, 1994.

15. S. Lattemann and T. Höpner, Seawater desalination: impacts of brine and chemical discharge on the marine environment, Balaban Desalination Publications, ISBN: 0866890629, Italy, 2003.

16. F. Van der Ham, G. J. Witkamp, J. de Graauw, G. M. van Rosmalen, Eutectic freeze crystallization: Application to process streams and waste water purification, Chem. Eng. Process., 37 (2) (1998) 207-213.

17. E. R. Pounder, Physics of ice, Pergamon Press Ltd., Library of Congress Catalogue Card No. 65-21141, 1965.

18. E. El-Sayed, and Y. El-Sayed, The advantages and the challenges of zero liquid discharge desalination. In: $3^{\text {rd }}$ International Conference on Desalination Technologies, Sharm El -Sheikh, Egypt, May 7 - 8, 2007, Conference Proceedings. 